15 | 1993

Varia

\title{
Le dialogue avec Pascal
}

Proust - Péguy - Valéry

Annie Barnes

\section{OpenEdition}

\section{Journals}

Édition électronique

URL : http://journals.openedition.org/ccibp/603

DOI : $10.4000 /$ ccibp.603

ISSN : 2493-7460

Éditeur

Centre international Blaise Pascal

\section{Édition imprimée}

Date de publication : 27 février 1993

Pagination : 17-42

ISSN : 0249-6674

Référence électronique

Annie Barnes, « Le dialogue avec Pascal », Courrier du Centre international Blaise Pascal [En ligne], 15 | 1993, mis en ligne le 07 janvier 2016, consulté le 20 avril 2019. URL : http:// journals.openedition.org/ccibp/603 ; DOI : 10.4000/ccibp.603

Ce document a été généré automatiquement le 20 avril 2019

Centre international Blaise Pascal 


\title{
Le dialogue avec Pascal
}

\author{
Proust - Péguy - Valéry
}

\section{Annie Barnes}

\section{Le Dialogue de Pascal}

1 Le dialogue avec Pascal a commencé de son vivant et le dialogue avec l'auteur des Pensées a commencé dès la publication de l'édition de Port-Royal. Il s'est poursuivi jusqu'à nos jours.

2 Faut-il rappeler que la première critique des Pensées, celle de l'abbé Villars en 1671, se trouve dans un entretien, - le cinquième des dialogues du Traité de la délicatesse, - entre Paschase, Ménippe qui l'admire servilement, et Aliton, un « Alceste moliniste » (Bernard Amoudru) : «Pauvre Paschase, vous serez malade toute votre vie. »? Faut-il rappeler que la meilleure réponse aux Provinciales, un peu tardive il est vrai, est celle du Père Daniel dans les Entretiens de Cléandre et d'Eudoxe? L'Anti-Pascal de Voltaire, dans la $25^{\mathrm{e}}$ des Lettres philosophiques, se présentera, ou plutôt fera semblant de se présenter, comme une suite d'articles sagement numérotés, une réfutation en forme. Mais dès le $3^{e}$ article, Voltaire entre en dialogue : « Qu'aurait répondu M. Pascal à un homme qui lui aurait dit... » Et l'on se souvient de la lettre à d'Argental (mai 1734) : « Va, va, Pascal, laisse-moi faire! Tu as un chapitre sur les prophéties où il n'y a pas l'ombre de bon sens ; attends, attends ! »

Dans les pages de son Port-Royal où il expose les dangers de Tartuffe, Sainte-Beuve imagine un dialogue entre Pascal et Molière. Et hier encore, que l'abbé Steinmann veuille se faire l'apologiste de l'oratorien Richard Simon, fondateur de l'exégèse biblique moderne, dénoncé si violemment par Bossuet, il le fait non seulement dans son ouvrage sur Richard Simon. Douze ans plus tôt, il publiait anonymement dans la Vie intellectuelle, la revue dominicaine, (1949) un Entretien de Pascal et du Père Richard Simon sur le sens de l'Écriture, qui est un pastiche très malicieux de Nicolas Fontaine, et qui « eut le don de provoquer l'ire de Paul Claudel », nouveau Bossuet.

4 Ou que Charles Maurras, dans sa prison, relise les Pensées, il composera un Conte infernal, Pascal puni, dont la majeure partie sera un nouvel entretien de Pascal avec M. de Sacy. Ou 
encore, que Jean Pommier veuille se délasser de ses travaux érudits sur la Jeunesse cléricale d'Ernest Renan, il composera un Dialogue de Renan et de Pascal dans l'au-delà (1923).

Si Pascal appelle le dialogue c'est, n'en doutons pas, précisément parce qu'il est lui-même homme de dialogue. Faudrait-il plutôt dire, - car souvent la personne à qui Pascal s'adresse reste invisible, muette, parfois on a même peine à l'identifier - faudrait-il plutôt dire que Pascal toujours parle à quelqu'un, qu'il est une voix ? Cela est vrai non seulement de l'adversaire des Jésuites ou de l'apologiste de la religion chrétienne; qu'il s'agisse même du géomètre ou du savant, toujours Pascal s'adresse à quelqu'un.

Dès son premier écrit, le Traité sur les Coniques composé quand Pascal à seize ans, paraît comme un refrain : "nous démontrerons ", « nous énoncerons ", " nous démontrerons ", paraît un moi juvénile et pas du tout haïssable : « ... et veux bien avouer que je dois le peu que j'ai trouvé en cette matière en ses écrits... ». C'est de M. Desargues qu'il est question, du mathématicien Desargues que nous retrouverons dans les Pensées, représenté par les raisins de Condrieu ${ }^{1}$. Cinq ans plus tard, la machine arithmétique sera accompagnée d'un Avis nécessaire, qui interpelle l'" Ami lecteur" et d'une Lettre dédicatoire au chancelier Séguier, en attendant la magnifique Lettre à la reine Christine de Suède. En octobre 1647 Les Expériences nouvelles touchant le vide commencent aussi par s'adresser à "mon cher lecteur ", et seront suivies d'une correspondance moins amicale avec celui qui ne s'était pas montré digne de ce titre, le Très Révérend Père Noël. Un an plus tard, le Récit de la grande expérience de l'équilibre des liqueurs se présente sous un aspect encore plus personnel. Pascal y publie la lettre à son beau-frère, Florin Périer, le priant de faire l'expérience sur le Puy-de-Dôme, il publie la réponse de Périer qui contient le récit de la grande expérience, et tout cela est relié par un commentaire à la première personne où Pascal finit par s'adresser de nouveau à «mon cher lecteur». On croirait presque lire un reportage d'aujourd'hui.

7 Après la conversation de novembre 1654, et grâce à elle, le géomètre et le savant cèdent le pas au défenseur de la religion, et Pascal devient le Pascal prince au royaume des lettres, un royaume qui ne l'ignore pas. Le dialogue de Pascal se poursuit alors sur un autre terrain. Sans parler de l'Entretien avec M. de Sacy, qui est sans doute fictif, bien que s'y entende indubitablement la voix de Pascal s'adressant à Sacy, les Provinciales ne comprennent-elles pas toutes les formes possibles de ce que nous entendons par le mot dialogue? «Et vous semble-t-il que les Lettres Provinciales soient autre chose que des comédies? " dira méchamment Racine à Nicole. Cela n'est vrai sans doute que des premières. Mais quand le ton devient grave et que Pascal renoncera à s'adresser à son provincial fictif, ce sera pour s'adresser directement aux Jésuites, d'abord à Mes Révérends Pères, de la onzième à la seizième lettre, enfin à Mon Révérend Père, - le Père Annat -, dans les deux dernières.

outes ces formes se trouvent également dans les Pensées, bien que souvent à l'état embryonnaire. La première des liasses titrées, la liasse Ordre, contient des projets de lettres, et des projets de dialogues: "Ordre par dialogues" ", "Lettre pour porter à rechercher Dieu ${ }^{3}$ »... sur les douze fragments qui composent cette liasse, sept ont l'une ou l'autre de ces indications.

9 Des vingt-six liasses titrées qui suivent, de Vanité à Conclusion, vingt-deux contiennent des fragments qui sont des esquisses de dialogues, soit qu'on y trouve question et réponse, ou bribe de conversation comme dans deux fragments sur le Divertissement (S. 165 et 171/ L. 132 et 139), ou encore, dans Commencement, le fragment S. 190 / L. 158 : «Par les partis vous devez vous mettre en peine de rechercher la vérité car si vous mourez sans adorer le 
vrai principe vous êtes perdu. Mais - dites-vous... ", soit que Pascal fasse parler la Sagesse de Dieu, soit qu'il interpelle brusquement un vous invisible et non identifié, soit qu'il parle à la première personne du singulier ou du pluriel. Ce dernier cas est le plus fréquent et ne va pas sans créer des difficultés d'interprétation sur lesquelles il nous faudra revenir. Et Pascal trouve encore d'autres moyens d'imposer sa présence, par une interrogation soudaine, par un Qui ou un Pourquoi, ou par une exclamation introduite par Quel ou Que. Il parvient même à donner un relief saisissant au pronom le plus incolore de sa nature : on. Ainsi ${ }^{4}$, de l'hypothèse des apôtres fourbes, il dira : «Qu'on la suive tout au long... » et pour terminer il répète : "Qu'on suive cela ». Si des liasses titrées, nous passons aux trentequatre séries, nous retrouvons tous ces caractères de style. Enfin, si nous lisons les fragments personnels que seul le Recueil Original nous a conservés, qu'y trouvons-nous sinon le dialogue de Pascal avec Dieu, dans le Mystère de Jésus ${ }^{5}$ ?

M. Jean Demorest à qui nous devons d'excellentes pages sur cet emploi des pronoms chez Pascal, écrit :

Cette même recherche d'un dynamisme d'essence oblige Pascal à poser son œuvre en termes polémiques ou apologétiques. Il faut qu'il agisse, qu'il persuade, qu'il lutte et convainque. Il se défend, il riposte, il a toujours affaire soit à un jésuite soit à un incroyant.

11 Quand Pascal a affaire à un jésuite, nous laissons au jésuite le soin de riposter, mais quand il a affaire à un incroyant, c'est tout autre chose. Le lecteur des Pensées se sent personnellement visé, et suivant son tempérament plus ou moins combatif et selon son attitude à l'égard du problème, ou faut-il dire, ce qui dirait peut-être Pascal, selon l'état de son âme, lui aussi ripostera. Comment rester indifférent en face d'un auteur toujours impérieux, souvent irrité et impatient, et ironique surtout, ironique un peu trop souvent pour le bien de sa cause ? Et voilà bien pourquoi il y a depuis trois cents ans un dialogue avec Pascal. Voilà aussi pourquoi ce dialogue est essentiellement un dialogue avec l'auteur des Pensées plutôt qu'avec l'auteur des Provinciales, ce qui n'empêchera pas cellesci d'exercer quelquefois une influence littéraire, comme nous le verrons surtout en parlant de Péguy.

12 Dans ce dialogue avec Pascal, trois écrivains majeurs vont retenir notre attention: Proust, Péguy, Valéry. Ce choix a été guidé par des préférences personnelles et l'on ne peut guère imaginer de prime abord un trio plus disparate. Ils ont cependant quelque chose en commun qui a son importance : ils appartiennent à la même génération. Le 10 juillet 1871, naissance à Paris de Marcel Proust ; le 30 octobre de la même année, naissance à Sète de Paul Valéry ; le 7 janvier 1873, naissance de Charles Péguy à Orléans : entre l'aîné et le plus jeune il n'y a que dix-huit mois. Malgré les très grandes différences de leurs milieux respectifs, de leurs esprits et de leurs tempéraments, ils ont donc grandi dans le même climat intellectuel et spirituel, ils ont été formés par le même enseignement officiel, celui des lycées de la République laïque sinon anti-cléricale. À l'époque de leur adolescence, il est vrai, commence à se dessiner nettement une réaction idéaliste qui trouvera l'une de ses expressions, après 1890, par ce que Margaret Eastwood, dans une belle thèse d'Oxford, a intitulé The Revival of Pascal.

13 Mais ce qu'il importe de rappeler ici, c'est que nos trois écrivains ont étudié Pascal dans leur année de rhétorique, et qu'ils ont lu les Pensées dans la même édition, qui est l'édition classique de Havet.

La première édition de Havet a paru en 1852. Havet en a tiré une édition abrégée pour les classes avant 1865 et c'est donc cette « édition classique » qu'il faut examiner pour savoir 
comment Pascal s'est présenté à nos trois lycéens. En voici le titre complet: Pensées de Pascal publiées dans leur texte authentique avec un commentaire suivi par Ernest Havet.

L'érudition de Havet ne s'est pas exercée sur le texte: il l'a pris tel quel dans l'édition Faugère (1844), la première édition qui, à l'instigation de Victor Cousin, est revenue aux autographes du Recueil Original. L'édition ne se présente pas non plus dans un ordre nouveau: Havet s'est contenté de suivre l'ordre de l'édition de Bossut de 1779, avec de légères modifications, et son édition répartit les fragments des Pensées en vingt-quatre articles, comme Bossut, en y ajoutant un vingt-cinquième qui contient les fragments publiés pour la première fois par Cousin ou par Faugère. Quant au texte, c'est seulement ici qu'on peut parler d'une édition abrégée pour les classes, ce dernier article ne contenant que 115 fragments, tandis que la grande édition Havet en contient 208. Cet article est suivi du Mystère de Jésus, publié pour la première fois, on s'en souvient, par Faugère. Nos lycéens des années quatre-vingt ont pu faire une étude des Pensées aussi sérieuse que la génération suivante avec l'édition que nous avons tous connue sous le nom de " petit Brunschvicg ", c'est à dire les Pensées et opuscules du petit volume Hachette. Car Havet lui aussi ajoute la plupart des opuscules de Pascal à son édition classique des Pensées : L'Entretien avec M. de Sacy, et la Prière pour le bon usage des maladies, et le Fragment d'un traité du vide, et De l'esprit géométrique, Sur la conversion du pécheur, Comparaison des chrétiens des premiers temps avec ceux d'aujourd'hui, la Lettre sur la mort de M. Pascal le père, et des extraits de lettres à $M^{\text {lle }}$ de Roannez.

L'apport personnel de Havet, et la preuve de son érudition, se trouvent dans son commentaire suivi. Nous lui devons la découverte d'une très grande quantité des sources de Pascal, et ses références ont été reprises par tous les éditeurs des Pensées, à commencer par Brunschvicg. Mais surtout il s'attache à expliquer les obscurités, à aplanir les difficultés d'interprétation, à faire ressortir la valeur des arguments ou, très souvent, la beauté du style. Et il fait des rapprochements fréquents avec des fragments placés dans des articles parfois très éloignés de celui qu'il commente, facilitant ainsi grandement une étude sérieuse des Pensées.

Dans quel esprit ce commentaire a-t-il été écrit? Péguy citera un critique contemporain qui parle du «très libéral Ernest Havet ». Il me semble que cela le décrit assez bien. Son admiration pour le savant est sans limite, et il admire presque autant l'écrivain comme maître en éloquence. Quant à l'apologiste, il essaie de l'apprécier objectivement, et il voit en lui surtout un sceptique et un janséniste. Il est évident qu'il ne partage ni sa foi ni son zèle pour la religion. Mais il est également exempt de tout zèle anticlérical, et il reprochera par exemple à Condorcet d'avoir supprimé presque en entier le fragment sur les «fleuves de Babylone ${ }^{6}$ » et tout le fragment sur les «trois ordres ${ }^{7}$ ». Si Havet n'a certainement pas essayé de montrer sa grandeur en étant à l'une ou l'autre extrémité, il ne réussit pas non plus à remplir l'entre-deux ${ }^{8}$. Mais est-il possible de ne pas prendre parti, surtout quand il s'agit de Pascal ? Je ne sais trop dans quelle catégorie placer Ernest Havet, qui ne me semble pas «chercher en gémissant », mais je ne puis m'empêcher de signaler l'effet qu'a produit son édition sur un autre Ernest, plus grand que lui bien que son cadet de dix ans. Le 6 juillet 1852, Renan écrit à Havet :

On voit partout, dans ces fragments, un esprit inexpérimenté dans les sciences historiques et critiques... Je crois pour ma part que le livre de Pascal, s'il l'eût mené à fin, eût été de tout point faux et insupportable. L'essentiel eût été vaine argumentation par les prophètes et les miracles... Vous relevez avec raison tout ce que cette argumentation a de puéril et sur quelle pauvre érudition elle repose... 

se présentent, et qui est donc celui de Bossut. Il est évident que cet ordre à une très grande importance pour qui veut juger de l'effet produit sur le lecteur. projetée avec les matériaux des Pensées, et bien que ces articles aient des titres dans le genre de ceux de l'édition de Port-Royal, mais que Havet n'a pas reproduits dans son édition pour les classes, il place le plus souvent en tête d'un article, un fragment particulièrement important par son contenu, sa longueur, son style achevé. C'est ainsi que le lecteur commence sa lecture des Pensées par le fameux :

...Que l'homme contemple donc la nature entière dans sa haute et pleine majesté'...

C'est le fragment Disproportion de l'homme, titre authentique que Havet donne en note, mais qui trop souvent aujourd'hui encore est nommé «les deux infinis». Pascal, lui, l'avait enfilé dans sa quinzième liasse, Transition de la connaissance de l'homme à Dieu. Ou bien l'article IX met en première place cette sorte de préface que la première Copie a enregistré dans sa Série III, qui débute par cette brusque entrée en matière, cet impérieux "...Qu'ils apprennent au moins quelle est la religion qu'ils combattent, avant que de combattre ", et qui contient quelques pages plus loin ce qu'on appelle «le monologue de l'athée ». Le premier fragment de l'article suivant, l'article X, n'est autre que le plus célèbre sans doute des fragments des Pensées, faussement appelé « le pari ». Havet, il est vrai, ne lui donne pas ce titre populaire, mais il se contente d'indiquer en note le vrai titre: Infini-Rien. Enfin, c'est à l'article XVIII, de nouveau en première place, que les lycéens des années quatre-vingts ont pu lire notre fragment S. 339 / L. 308 que Pascal a enfilé dans a liasse 23, Preuves de Jésus-Christ :

La distance infinie des corps aux esprits figure la distance infiniment plus infinie des esprits à la charité, car elle est surnaturelle...

que nous appelons communément le fragment « des trois ordres ». Havet lui consacre de très nombreuses notes, surtout pour expliquer les obscurités de la syntaxe, « car », dit-il, «tout cela est écrit très négligemment». C'est ainsi qu'il parle de ce que de très nombreux lecteurs de Pascal ont considéré et continueront à considérer comme le plus beau texte des Pensées.

Résumons nos observations sur l'édition Havet pour les classes: aucune invitation à spéculer ce qu'aurait pu être l'Apologie de Pascal, à se préoccuper de questions d'ordre ; une invitation à voir en Pascal un sceptique pour qui seul « le pyrrhonisme est le vrai ${ }^{10}$ »; enfin, par le seul fait que certains grands textes, ceux que je viens d'énumérer, sont mis en vedette, une invitation à concentrer son attention sur certains problèmes et sur certaines notions pascaliennes: la notion des trois ordres d'une part, celle du pari de l'autre, enfin ce qu'on a si souvent appelé « l'angoisse de Pascal».

La notion des trois ordres ne nous retiendra pas ici, puisque ce chapitre est censé être consacré au dialogue de Pascal plutôt qu'au dialogue avec Pascal, et nous laisseront surtout à Péguy le soin de commenter le fragment sur « la distance infinie ». avec les deux autres problèmes, nous revenons à notre sujet, et avant de rechercher de quelle manière nos trois auteurs ont compris ou discuté l'argument du pari ou l'angoisse de Pascal, je voudrais rappeler comment nous voyons ces problèmes, aujourd'hui que grâce aux progrès des études pascaliennes depuis vingt ans, nous pouvons nous appuyer fermement sur le classement des Pensées que nous ont conservé la première et la deuxième Copie, et

Courrier du Centre international Blaise Pascal, 15 | 2015 
que reproduisent les dernières éditions Lafuma, celle dite des manuscrits, celle du Livre de vie, celle de la collection L'Intégrale, et celle de Philippe Sellier.

Le texte Infini-rien, Pascal ne l'a pas classé parmi les 27 liasses à titre où il a enfilé 382 fragments. Il se trouve parmi les papiers qu'on appelle "non classés", distribués en 34 Séries, dans la seconde partie de la première Copie, et forme avec toutes ses additions marginales la Série II. Nous ne nous proposons pas ici une analyse ni une discussion de ce texte dont Jean Orcibal a dit: "Il n'y a guère de page des Pensées qui présente des difficultés plus graves et soit, au point de vue littéraire, plus indigne de l'auteur des Provinciales». Renvoyons surtout au livre indispensable de Georges Brunet, Le pari de Pascal, pour ne rappeler ici que quelques points d'une importance capitale au sujet de cette étude. Le texte peut se diviser en quatre parties, de longueur très inégale. Dans une espèce de préambule où il parle à la première personne du pluriel, Pascal nous conduit à admettre que par la raison nous sommes incapables de connaitre ce que Dieu est, ni s'il est. Alors le dialogue s'engage, et l'apologiste invite le libertin à parier que Dieu est, parce que c'est dans son intérêt, tout en soulignant la nécessité d'un choix : "Cela n'est pas volontaire, vous êtes embarqué.» À la fin de cette deuxième partie, où l'autographe montre des additions qui ajoutent grandement aux difficultés d'interprétation, mais qui ne nous arrêteront pas, le libertin admet qu'il faut parier, et parier pour Dieu, mais en déclarant qu'il «est fait de telle sorte qu'il ne peut croire. " Suivent alors des conseils pratiques pour diminuer les passions « qui sont vos grands obstacles », « prendre de l'eau bénite et faire dire des messes », ce qui conduit au fameux «Cela vous abêtira». Enfin, dans une dernière partie, très courte, intitulée Fin de ce discours, le dialogue se termine par l'exclamation « $O$ ce discours me transporte, me ravit» et par la réponse de l'apologiste, de l'homme qui s'est mis à genoux pour demander à Dieu la conversion du libertin.

24 Ceci ressort avec évidence, de ce résumé sommaire, et c'est pourquoi il est vain, presque absurde, de tenter de classer Infini-rien parmi les liasses titrées, ou de la placer dans l'apologie projetée. Infini-rien restera à tout jamais inclassable parce qu'il est à lui seul une apologie complète, ou sans doute vaudrait-il mieux dire, un itinéraire complet de l'incroyance à la foi : au départ, un libertin, à l'arrivée, un homme converti.

Or, que représentent les 27 liasses titrées de la Copie sinon également un itinéraire de l'incroyance à la foi, - si nous les considérons du point de vue de l'apologiste lui-même, mais évidemment sur une plus grande échelle ? Les conclusions de M. Brunet sont encore plus passionnantes que son analyse du texte. Différents indices le conduisent à penser que Infini-rien a été écrit dès septembre 1656, en pleine bataille des Provinciales, et que c'est donc le texte apologétique de Pascal le plus ancien que nous possédions. Ensuite, par de nombreux rapprochements avec les Pensées, il montre avec évidence que toutes les notions que contenait ce «brouillon griffonné à la diable » ont été reprises par Pascal et approfondies, souvent enfilées dans les liasses à titre, - toutes, à l'exception d'une seule : ce qu'on pourrait appeler le pari proprement dit, d'une part la proposition de jouer l'existence de Dieu à pile ou face (« cet article paraît un peu indécent et puéril » comme dit Voltaire), d'autre part cette suite d'hypothèses sur les chances de gain qui font croire qu'on a affaire à un calcul de probabilités, - tout cela, Pascal l'a laissé tomber pour ne plus jamais le reprendre.

26 C'est pourquoi nous le laissons tomber nous aussi, pour nous attacher à mieux comprendre le sens réel du "pari ». C'est une notion complexe qui implique d'abord un choix, un choix qui est nécessaire, inévitable, qui engage toute la personne, et qui 
comporte un risque, - le risque évidemment d'avoir fait le mauvais choix (mais ce risque est tout subjectif et ne joue qu'avant le choix qu'est la conversion : l'apologiste, lui, sait "que vous connaîtrez à la fin que vous avez parié pour une chose certaine, infinie, pour laquelle vous n'avez rien donné »). De tous les aspects de cette notion complexe, je vois par expérience - l'expérience de longues années d'enseignement de Pascal - que le plus difficile à comprendre, c'est celui de la nécessité du "pari». Ici, Port-Royal avait parfaitement bien compris Pascal, car après le "Oui, il faut parier; cela n'est pas volontaire ; vous êtes embarqué » l'édition de 1670 avait ajouté cette glose : « et ne parier point que Dieu est, c'est parier qu'il n'est pas, car celui qui doute et demande à s'éclairer ne parie assurément ni pour ni contre ». (Comme je ne puis m'empêcher d'avoir de l'affection pour ce vieux Voltaire, je vous fais remarquer qu'il n'a pu s'empêcher d'ajouter: "et demande à s'éclairer». Ne serait-ce pas déjà parier pour?) Les existentialistes ont mieux compris Pascal. « Le choix est possible dans un sens, mais ce qui n'est pas possible, c'est de ne pas choisir ", dit Sartre. «Je peux toujours choisir, mais je dois savoir que si je ne choisis pas, je choisis encore.»

Sur ce point de la nécessité du pari, Havet a des notes très utiles et très sensées. Il ne cite pas la glose de Port-Royal, mais il cite l'Évangile : Qui non est mecum contra me est ${ }^{11}$, ce qui pourrait bien être la source biblique de Pascal, car il cite ce texte ailleurs.

Si Infini-rien est écrit sous forme de dialogue, ce n'est pas cela qui rend l'interprétation difficile, bien que les critiques ne soient pas toujours d'accord sur ce qu'il faut mettre dans la bouche de l'apologiste ou dans celle du libertin. Mais si nous passons au sujet de l'« angoisse de Pascal », il n'en va plus de même. On pourrait aller jusqu'à prétendre que ce sujet riche entre tous par les commentaires qu'il a suscités, n'existe que parce que Pascal a utilisé le dialogue sans se donner la peine de nous en avertir, ou faut-il dire, sans crier gare.

Je crois bien que toute l'évidence de la soi-disant angoisse de Pascal consiste en cinq ou six textes, toujours les mêmes, qui fournissent le point de départ des commentaires, quels qu'ils soient. Énumérons-les; citons-les. Le plus court est aussi le plus célèbre :

Le silence éternel de ces espaces infinis m'effraie ${ }^{12}$.

Quand je considère la petite durée de ma vie absorbée dans l'éternité précédente et suivante -memoria hospitis unius diei praetereuntis - le petit espace que je remplis et même que je vois abîmé dans l'infinie immensité des espaces que j'ignore et qui m'ignorent, je m'effraie et m'étonne de me vois ici plutôt que là, car il n'y a point de raison pourquoi ici plutôt que là, pourquoi à présent plutôt que lors. Qui m'y a mis ? Par l'ordre et la conduite de qui ce lieu et ce temps a-t-il été destiné à moi ${ }^{13}$ ?

Le fragment S. 227 / L. 194 pose des questions semblables sinon tout aussi angoissées :

Pourquoi ma connaissance est-elle bornée, ma taille, ma durée à 100 plutôt qu'à 1000 ? Quelle raison a eu la nature de me la donner telle et de choisir ce milieu plutôt qu'un autre dans l'infinité, desquels il n'y a pas plus de raison de choisir l'un que l'autre, rien ne tentant plus que l'autre?

Le fragment S. 229 / L. 198 est plus long et d'un style remarquablement achevé, et je n'en cite que la première moitié, car le reste à un caractère tout différent :

En voyant l'aveuglement et la misère de l'homme, en regardant tout l'univers muet et l'homme sans lumière abandonné à lui-même, et comme égaré dans ce recoin de l'univers sans savoir qui l'y a mis, ce qu'il y est venu faire, ce qu'il deviendra en mourant, incapable de toute connaissance, j'entre en effroi comme un homme qu'on aurait porté endormi dans une île déserte et effroyable, et qui s'éveillerait sans connaitre et sans moyen d'en sortir. Et sur cela j'admire comme on n'entre point en désespoir d'un si misérable état... 

surtout ses lettres personnelles où nous ne trouvons pas l'ombre d'effroi devant les espaces infinis, et en revanche une claire allusion à la doctrine biblique selon laquelle la nature visible reflète un Dieu invisible ${ }^{15}$, comme dans le Psaume 18 : Les cieux racontent la gloire de Dieu. Ensuite il a fallu oublier le dernier des textes que j'ai cités, où Pascal fait exprimer au libertin lui-même ces sentiments d'effroi, où la question du « Qui parle? » ne se pose même pas, du moins à un niveau superficiel (Ce monologue de l'athée est fort complexe et nous y reviendrons). C'est ainsi que par le rapprochement de ces cinq ou six textes, donc par la seule évidence interne, Jeanne Russier a pu conclure que partout c'est le libertin qui parle et non Pascal. Une de ses observations me semble particulièrement importante, c'est que la citation latine du fragment 68: memoria hospitis unius diei praetereuntis, Pascal l'a trouvée à la fin d'un verset du livre de la Sagesse ${ }^{16}$, et voici le verset entier :

Oui l'espoir de l'impie est comme balle emportée par le vent, comme écume légère qui chasse la tempête ;

il se dissipe comme fumée au vent, il s'efface comme le souvenir de l'hôte d'un jour. C'est donc de l'impie qu'il s'agit, c'est lui qui a inspiré à Pascal ces questions.

Jeanne Russier a utilisé l'édition Brunschvicg pour son ouvrage La foi selon Pascal. Aujourd'hui que nous pouvons lire les Pensées telles qu'elles ont été classées par Pascal, la notion d'un Pascal effrayé devant les espaces infinis est devenue encore plus absurde.

C'est un homme éminemment pratique que Pascal. Après tout, le tricentenaire de sa mort ne l'a-t-il pas célébré, entre autres, comme l'ancêtre de nos transports publics, et de nos ordinateurs? L'auteur des Provinciales a un but essentiellement pratique : défendre PortRoyal, discréditer les Jésuites. Les Pensées de même ont un but pratique. Bien plus qu'écrire une apologie, c'est-à-dire une Défense de la religion chrétienne comme par 
exemple l'avait fait Grotius, Pascal voulait convertir des hommes "concrets", comme Méré et Miton, et l'évidence des liasses titrées nous montre clairement que l'ouvrage projeté aurait été une puissante machine à conversion, pour ainsi dire. Or, que voyonsnous? Pascal a enfilé le fragment qui décrit l'impie de la Bible dans sa liasse Misère, et trois autres fragments à la première personne ${ }^{17}$ dans la liasse Transition de la connaissance de l'homme à Dieu. Qu'est-ce à dire ? Que pour continuer à soutenir que Pascal a exprimé un effroi personnel, il faut s'imaginer un homme qui construit soigneusement sa machine à convertir, cet itinéraire de l'incroyance à la foi, mais qui ne peut s'empêcher ici et là d'enfiler un petit bout de papier, qu'il découpe d'abord soigneusement, où il a jeté, sans doute pendant une nuit d'insomnie, l'aveu d'une terrible angoisse personnelle. Pascal évidemment souffre de schizophrénie avancée... ept références à Pascal, et qui se trouvent pour la plupart dans les notes. Donc peu ou pas d'influence littéraire de Pascal sur Proust. Une influence religieuse semble encore moins probable. En effet, nombreux sont les critiques qui refusent à Proust tout sens

Courrier du Centre international Blaise Pascal, 15 | 2015 
religieux. Le 12 janvier 1962, après avoir écouté à la T.S.F. le reportage sur Proust, Julien Green note dans son Journal :

Repensé à l'émission sur Proust. Il ne s'est pas trouvé un chrétien pour dire que dans l'œuvre de Proust, que l'on considère parfois comme le plus grand événement de ce siècle Dieu est absent, et absent d'une façon éclatante. Il y a, je le sais, la phrase sur le petit mur de Vermeer : c'est peu.

Banquet l'article L'irréligion d'État signé Laurence. L'auteur s'y présente comme défenseur de la religion chrétienne. En plus de ce sujet tout pascalien, dans ce texte d'à peine deux pages, il trouve moyen de faire allusion à un opuscule de Pascal, il donne Pascal comme exemple, avec Descartes, d'une discipline religieuse qui ne gêne pas le libre génie, et il utilise la notion de pari d'une façon toute pascalienne. Voyez plutôt :

Aussi bien un enseignement qui n'est pas religieux n'est-il pas forcément athéiste? Ne pas prendre parti sur Dieu, sur l'âme, quand il s'agit d'un enseignement tout entier, n'est-ce pas encore une façon, et la pire, de prendre parti ?

Voilà pour le pari. On croit entendre l'écho du texte des Pensées dans l'édition de PortRoyal : « ...et ne parier point que Dieu est, c'est parier qu'il n'est pas ». Havet ne cite pas cette glose de Port-Royal dans ses notes, mais en revanche il cite un texte de l'Évangile 
qui a pu inspirer Proust: Qui non est mecum contra me est ${ }^{19}$. Et voici l'allusion à un autre texte pascalien :

Eh bien, cette théorie de la vie et du bonheur (celle qu'enseigne le christianisme) existe, elle a été longtemps acceptée et a juste titre ; elle est vraie, d'une vérité, il faut bien le dire, qui a contre elle d'être méconnue des rédacteurs de l'Intransigeant et de la Lanterne (mais il n'y a pas d'autorité en matière de philosophie !). première fois en 1779, au fragment de Préface sur le Traité du vide où Pascal attaque précisément l'autorité des anciens en matière de philosophie c'est-à-dire dans les sciences physiques; l'allusion est d'autant plus piquante que l'ironie de la phrase proustienne rappelle celle des Provinciales. Mais continuons à citer ce texte remarquable pour arriver au passage qui mentionne Pascal :

C'est à des esprits comme élevés au-dessus d'eux-mêmes par le Christianisme que la France doit ses plus purs chefs-d'œuvre, soit dans le domaine de l'action, soit dans le domaine de la spéculation. Aujourd'hui encore, tandis que les missionnaires français civilisent l'Orient, le plus hardi philosophe de ce temps pourrait scandaliser l'épicier matérialiste du coin par sa piété rigoureuse. Cette discipline religieuse à laquelle il se soumet, et qui ne gênait ni Descartes, ni Pascal, serait une entrave parait-il, pour le libre génie de certains conseillers municipaux...

\section{grande sommé Á la recherche du temps perdu. Des sept passages auxquels réfère l'index de} la Pléiade, citons les quatre premiers. visite de Swann. Dans sa conversation avec le grand-père et les tantes du narrateur, Swann parle des « assommants journaux »;

Ce que je reproche aux journaux, c'est de nous faire faire attention tous les jours à des choses insignifiantes, tandis que nous lisons trois ou quatre fois dans notre vie les livres où il $\mathrm{y}$ a des choses essentielles. Du moment que nous déchirons fiévreusement chaque matin la bande du journal, alors on devrait changer les choses et mettre dans le journal, moi je ne sais pas, les... Pensées de Pascal I (il détacha ce mot d'un ton d'emphase ironique pour ne pas avoir l'air pédant ${ }^{20}$ ).

Notre deuxième passage est pris du Côté de Guermantes. Françoise est en conversation avec le valet de chambre :

C'est une grande famille que les Guermantes ! ajoutait-elle avec respect, fondant la grandeur de cette famille à la fois sur le nombre de ses membres et l'éclat de son illustration, comme Pascal, la vérité de la Religion sur la Raison et l'autorité des écritures. Car n'ayant que ce seul mot de "grand" pour les deux choses, il lui semblait qu'elles n'en formaient qu'une seule ${ }^{21}$..

Nous voici maintenant, 700 pages plus loin, à la fin de la soirée chez la princesse de Guermantes. Basin invite Charlus à Guermantes en rappelant à son frère des souvenirs d'enfance :

Te rappelles-tu le vieux père Courveau : « Pourquoi est-ce que Pascal est troublant ? Parce qu'il est trou... trou...blé », prononça M. de Charlus comme s'il répondait encore à son professeur. - «Et pourquoi est-ce que Pascal est troublé ? Parce qu'il est trou... parce qu'il est trou... - Blanc. - Très bien, vous serez reçu, vous aurez 
certainement une mention, et $\mathrm{M}^{\text {me }}$ la duchesse vous donnera un dictionnaire chinois ». Si je me rappelle, mon petit Méméz ${ }^{2} ! .$. de moraliste :

À partir d'un certain âge nos souvenirs sont tellement entrecroisés les uns sur les autres que la chose à laquelle on pense, le livre qu'on lit n'a presque plus d'importance. On a mis de soi-même partout, tout est fécond, tout est dangereux, et on peut faire d'aussi précieuses découvertes que dans les Pensées de Pascal dans une réclame de savon ${ }^{23}$.

61 Ces quatre passages ne voient en Pascal que l'auteur des Pensées, et le premier et le dernier donnent assurément aux Pensées de Pascal une place éminente, suréminente même parmi les livres. L'allusion à propos de Françoise, à la méthode apologétique de Pascal, montre bien que Proust n'a pas oublié ce qu'il a appris en rhétorique à Condorcet, et l'échange de souvenirs entre le duc de Guermantes et le baron de Charlus révèle en plus que la fameuse «angoisse de Pascal» fait partie intégrante de ses connaissances pascaliennes, quelque interprétation qu'il en ait donnée par ailleurs. Un passage de la Recherche $^{24}$ fait de même allusion à un autre lieu commun de l'époque au sujet de Pascal, à l'abîme de Pascal. Ici il faut sans doute voir une réminiscence de Baudelaire dans les Nouvelles Fleurs du mal :

Pascal avait son gouffre, avec lui se mouvant.

- Hélas ! tout est abîme, - action, désir, rêve...

Baudelaire auquel Proust a consacré tant d'admirables pages de critique, et surtout le chapitre du Contre Sainte-Beuve qui se termine par cette longue phrase sur le

visage de ce grand poète qui au fond est un, depuis le commencement du monde, dont la vie intermittente, aussi longue que celle de l'humanité eut en ce siècle ses heures tourmentées et cruelles, que nous appelons vie de Baudelaire, ses heures laborieuses et sereines, que nous appelons vie de Hugo, ses heures vagabondes et innocentes que nous appelons vie de Gérard et peut-être de Francis Jammes ses égarements et abaissements sur des buts d'ambition étrangers à la vérité, que nous appelons vie de Chateaubriand et de Balzac, ses égarements et surélévation audessus de la vérité, que nous appelons deuxième partie de la vie de Tolstoï, comme de Racine, de Pascal, de Ruskin, peut-être de Maeterlinck.

On notera l'antithèse très pascalienne d'abaissement - surélévation, renforcée par la symétrie de cette fin de phrase. Est-ce à dire que Proust croit aux égarements de Pascal pendant ce que la critique a longtemps appelé « la période mondaine », qui a précédé la conversion de novembre 1654 ? Je le suppose. Quant à l'expression «surélévation audessus de la vérité », elle est assez mystérieuse, et Pascal s'y trouve placé dans un groupe de noms bizarrement disparates... Ce qui reste certain, c'est que Pascal, pour Proust, fait partie du visage du grand poète qui au fond est un depuis le commencement du monde.

Admiration de l'auteur des Pensées, et familiarité avec les Pensées, j'en trouve encore deux indices dans la Recherche du temps perdu auxquels l'Index de la Pléiade ne donne pas de référence parce que le nom de Pascal n'y figure pas. Ils sont tous deux dans le Temps retrouvé, et le premier fait réflexion sur l'effet de la guerre sur les esprits :

À vrai dire, ce changement profond opéré par la guerre était en raison inverse de la valeur des esprits touchés, du moins à partir d'un certain degré. Tout en bas, les purs sots, les purs gens de plaisir, ne s'occupaient pas qu'il y eût la guerre. Mais tout en haut, ceux qui se sont fait une vie intérieure ambiante ont peu égard à l'importance des événements ${ }^{25}$... 
assage se trouve au milieu de la longue méditation du narrateur dans la bibliothèque du prince de Guermantes, dans ces pages où il s'élève contre l'« intelligence raisonneuse » de la critique, de ces " demi-esprits ", à mi-chemin entre le grand public et les esprits supérieurs, qui se trompent constamment sur la valeur des écrivains. Tout cela rappelle singulièrement les fragments que Pascal a classés dans sa liasse S. 6/L. 5, Raison des effets, ces réflexions sur les demi-habiles, sur la gradation des esprits ${ }^{26}$ et sur les opinions du peuple saines. Et comme pour indiquer que nous avons bien deviné, Proust termine ainsi son paragraphe ${ }^{27}$ en disant des « demi-esprits» :

ils agissent ainsi plus que les esprits supérieurs, attirent à eux la foule et créent autour d'eux non seulement les réputations surfaites et les dédains injustifiés, mais les guerres civiles et les guerres extérieures dont un peu de critique port-royaliste sur soi-même devrait préserver.

Familiarité avec les Pensées, nous en retrouvons des preuves nombreuses dans la Correspondance de Proust. Je me bornerai à quelques citations. Á Walter Berry, il écrit : «Vous êtes l'éloquence même, et aussi celle dont parle Pascal, qui se moque de l'éloquence ». Dans une lettre adressée à Reynaldo Hahn, le 6 août 1905, de Versailles, il pastiche $\mathrm{M}^{\mathrm{me}}$ de Sévigné puis La Fontaine pour raconter comment ayant regardé par la fenêtre de sa chambre à l'hôtel des Réservoirs, il n'a pu éviter un fâcheux, et en tire cette moralité : "Tout le malheur des hommes vient de ne pas se savoir renfermer en une chambre ». Mais les allusions à Pascal se rencontrent également sous sa plume quand il ne songe pas à plaisanter.

Les lettres de Proust au comte de Montesquiou, on le sait sont pleines de louanges hyperboliques. Est-ce parce que Robert de Montesquiou est l'auteur de Roseaux pensants que Proust mêle plusieurs fois Pascal à ses éloges du poète ? Ainsi dans la lettre XX, à propos de poèmes de Montesquiou parus dans la Revue de Paris, et après avoir comparé l'un d'eux à « une nuit de Goya », il continue :

Je pense que le portrait sans pitié de la « Miss » n'est pas placé là sans coquetterie et pour montrer que, comme Pascal, vous tenez tout l'entre-deux.

L'allusion est, on le voit, au fragment des Pensées où Pascal admire l'extrême valeur et l'extrême bénignité d'Épaminondas pour continuer par cette réflexion de moraliste :

On ne montre pas sa grandeur pour être à une extrémité, mais bien en touchant les deux à la fois, et remplissant tout l'entre-deux ${ }^{28}$.

Cette réflexion ne frappe guère dans l'édition Havet, où elle se trouve placée au fragment 21 de l'article VI. Je me demande si ce n'est pas Sainte-Beuve qui aurait attiré l'attention de Proust sur ce texte de Pascal, car dans son Port-Royal il en fait une application brillante à saint François de Sales ${ }^{29}$ et nous savons qu'en 1896 Proust a lu le Port-Royal de Sainte-Beuve qui a fait sur lui une impression durable. Ne parle-t-il pas dans la Recherche du temps perdu, comme nous venons de le voir, de « critique port-royaliste »?

Le plus grand nombre des lettres à Montesquiou, Proust les écrit dans son lit de malade, et voici une lettre qui remercie le comte d'une charmante soirée et continue :

Je souffre beaucoup ce soir et ne peux vous écrire plus longuement n'ayant pas cette admirable suprématie sur le corps que vous avez et qui fait penser à la fois à Pascal et à Sarah Bernhardt.

71 Il est permis de penser que Proust n'a pas fait ce rapprochement pour le moins inattendu sans un grain de malice. Mais voici encore une lettre à Montesquiou ou le doute n'est plus possible, Proust est sérieux ; il vient de perdre sa mère, et il remercie le comte de lui avoir écrit : 
Votre pitié dans ma détresse est une interprétation neuve et magnifique du « car la feuille de lis est tournée en dehors ». Et c'est dans ces moment-là plus qu'en aucun autre que vous êtes "plus splendide que Salomon dans toute sa gloire». Car «l'ordre de la charité est au-dessus de tous les autres». passage d'une lettre à son ami Antoine Bibesco qui doit être datée de 1901 :

Justement dans un Pascal et un La Bruyère dont je console ce soir mon regret, je trouve à diverses reprises le mot : ami...

La Bruyère dit : Un homme en place doit aimer les gens d'esprit, il les doit adopter... il ne saurait payer (je ne dis pas) de trop de bienfaits mais de trop de caresses... les leçons et les services qu'il en tire, même sans le savoir.

Quels petits bruits ne dissipent-ils pas (mais au moins me lisez-vous Bibesco, car si vous ne me lisez pas ce n'est pas la peine que je me tue à copier ce merveilleux passage) quelles histoires ne réduisent-ils pas à la fable et à la fiction (c'est trop long et je passe le plus beau) semer en mille occasions des faits et des détails qui soient avantageux et tourner le rire et la moquerie contre ceux qui avançaient des faits contraires, etc...

Et Pascal plus bref et plus fort (et que je me suis émerveillé de trouver si au courant de ce qui se passe chez $\mathrm{M}^{\text {me }}$ de Saint-V.) : un ami est une chose si avantageuse même pour les plus grands seigneurs, afin qu'il dise du bien d'eux et qu'il les soutienne en leur absence. Même (même est spirituel) qu'ils doivent tout faire pour en avoir. Mais qu'ils choisissent bien, car s'ils font tous leurs efforts pour des sots, cela leur sera inutile, etc... même s'ils (les sots) en disent du bien. Et même ils n'en diront pas du bien s'ils se trouvent les plus faibles, car ils n'ont pas d'autorité (sublime) et ils en médiront en compagnie.

J'aimerais (ce n'est plus Pascal qui parle et il est hélas superflu de vous en avertir) avoir de l'esprit et qu'il pût vous servir auprès des autres, mais vous n'êtes pas utilitaire et je ne suis pas utile...

Ce fragment des Pensées se trouve dans Havet à l'article VI. $55^{30}$, et si l'on s'y reporte, on découvre que dans une note Havet fait le rapprochement avec le texte de La Bruyère, pris du chapitre Des Grands, que Proust cite d'abord à Bibesco. Non que j'accuse Proust de mentir en disant « dans un Pascal et un La Bruyère dont je console ce soir mon regret »; au contraire, Proust certainement dit vrai, puisque sa citation de La Bruyère est plus longue que chez Havet, - mais l'idée même de lire l'un après l'autre ces deux textes ne viendrait-elle pas de la lecture de son Havet?

Et voici peut-être un autre indice de l'influence, du moins stylistique, de Pascal sur Proust. Dans une note à sa traduction de Sésame et les lys, il s'exclame : «Quelle vanité que la métaphore quand elle donne de la dignité à l'idée précisément à l'aide de fausses grandeurs dont nous nions la dignité». Cette phrase ne peut être autre chose qu'un pastiche conscient du fragment que Pascal a classé sous Vanité: «Quelle vanité que la peinture qui attire l'admiration par la ressemblance des choses dont on n'admire point les originaux $^{31}$ ! Faut-il voir en ce pastiche l'intention de se moquer un peu de Pascal en même temps que de Ruskin, puisque aussi bien ce fragment sur la vanité de la peinture, pris hors de son contexte, est un des plus discutables des Pensées? C'est possible. (Rappelons cependant que Pascal et Proust ont ceci en commun : ils rejettent tous deux la métaphore purement ornementale comme procédé stylistique). Mais le pastiche que Proust fait ici de Pascal peut s'interpréter d'une manière toute différente. Dans une lettre 
à la Princesse Bibesco, alors qu'il vient de lire le livre de la Princesse sur la Perse, Les huit paradis, Proust exprime son admiration. Il n'a que trois critiques à faire, dont voici la deuxième :

J'ai noté (mais je n'ai pas le livre sous la main) une ou deux phrases à la Barrès (qui sont peut-être des phrases à la Pascal : « Le... Quel... pour...»). Or je suis l'ennemi de tout pastiche, excepté quand il est voulu et encore!

Cette remarque peut sembler étrange venant de l'auteur de ces prestigieux Pastiches publiés dans Le Figaro sous le titre L'Affaire Lemoine. Voici comment on peut, je crois l'interpréter : nous savons que Proust s'est adonné à cet exercice littéraire un peu comme on prend un remède homéopathique. Rappelons cette page de son article sur Flaubert où, dit-il,

...pour ce qui concerne l'intoxication Flaubertienne, je ne saurais trop recommander aux écrivains la vertu purgative, exorcisante du pastiche.

Et un peu plus loin sur la même page :

Il faut... faire un pastiche volontaire, pour pouvoir après cela, redevenir original, ne pas faire toute sa vie du pastiche involontaire.

Certes, je ne veux pas prétendre qu'un pastiche de Pascal qui consiste en une seule phrase, soit la preuve que Proust cherchait à se débarrasser d'une "intoxication pascalienne ». Je me contente de conclure une fois de plus: Proust est familier avec les Pensées.

Sans décider s'il y a vraiment influence de Pascal sur Proust, ce qui me semble impossible d'établir avec certitude passons à l'autre aspect de notre sujet: peut-on signaler des parallèles entre Proust et Pascal, peut-on même parler d'affinités ? Ici il faut, me semble$\mathrm{t}$-il, répondre par l'affirmative. Essayons rapidement de voir pourquoi.

Le premier point de ressemblance est si évident qu'il est superflu de s'y arrêter. «Le Temps est le véritable héros de l'œuvre ", a-t-on dit de la Recherche du temps perdu. Ce temps perdu, l'écrivain Proust le recherche là seul où nous en ressentons vraiment le passage la fuite : en nous-mêmes. Le temps officiel, calculable, celui des horloges, dominé par la suite des jours et des nuits, est un temps qui nous reste extérieur. Or Pascal, comme Proust, est un être qui est profondément conscient de vivre dans le temps. "Vous êtes embarqué ». Image qu'il faut rapprocher du fragment ${ }^{32}:$ « Les fleuves de Babylone coulent et tombent, et entraînent... » et de bien d'autres encore. Voilà pourquoi on peut parler de temps humain à propos de Pascal et de Proust, et voilà pourquoi ils ont chacun leur chapitre dans le premier volume du bel ouvrage de Georges Poulet: Études sur le temps humain.

Mais le temps humain n'est pas un temps continu, et le cœur a ses intermittences. Les intermittences du cœur, rappelons-le, Proust pensait d'abord en faire le titre de son grand ouvrage, et il l'a gardé comme sous-titre d'une partie de Sodome et Gomorrhe II, celle où lors de son second séjour à Balbec, le narrateur éprouve soudain le chagrin de la mort de sa grand-mère. C'est ainsi que Proust est amené à faire ce qu'il appelle « une psychologie dans le temps ». Les thèmes principaux de cette psychologie dans le temps, on ne saurait les résumer mieux qu'en citant quelques Pensées de Pascal. Le fragment 698 débute par la question: «Qu'est-ce que le moi ?» Question essentielle, qui pourrait être aussi un titre de Proust. Le narrateur, dans ses interminables analyses de l'amour, de la mémoire, de l'oubli, pourrait ajouter à ses réflexions de moraliste ces deux fragments des Pensées : 
Le temps guérit les douleurs et les querelles parce qu'on change. On n'est plus la même personne, ni l'offensant, ni l'offensé, ne sont plus eux-mêmes ${ }^{33}$...

Il n'aime plus cette personne qu'il aimait il y a dix ans. Je crois bien : elle n'est plus la même ni lui non plus. Il était jeune et elle aussi elle est tout autre. Il l'aimerait peut-être encore telle qu'elle était alors ${ }^{34}$.

Et, dans une perspective un peu différente, voici la cinquième Pensée inédite découverte et publiée par Jean Mesnard :

Je me sens une malignité qui m'empêche de convenir de ce que dit Montaigne, que la vivacité et la fermeté s'affaiblissent en nous avec l'âge. Je ne voudrais pas que cela fût. Je me porte envie à moi-même. Ce moi de vingt ans n'est plus moi ${ }^{35}$.

Si Proust a fini par donner à sa grande œuvre le titre Á la recherche du temps perdu, il la résume par deux fois à l'intérieur de l'œuvre, quand le narrateur parle de « la vocation invisible dont cet ouvrage est l'histoire ». La vocation du narrateur aussi bien que celle de Proust, c'est d'écrire l'œuvre qui transformera le temps perdu en temps retrouvé. Et la vocation de Pascal ? À quoi est-il appelé en cette nuit du 23 novembre? Le papier du Mémorial nous le dira, où nous lisons :

«Oubli du monde et de tout hormis Dieu ».

Dans un sens, c'est la vocation de tout chrétien, tout simplement. À première vue, impossible d'établir un parallèle, bien plus: la seule pensée de trouver ici une ressemblance entre Pascal et Proust pourrait sembler choquante à certains. N'oublions pas l'observation de Julien Green : «Dans l'œuvre de Proust, Dieu est absent, et absent d'une façon éclatante. » Et je pense à certains critiques catholiques qui voient en Proust l'adepte d'une fausse religion, la religion de l'art, et un idolâtre de la Beauté. Pascal n'aurait sans doute pas approuvé Proust, ni dans sa vie, ni dans son œuvre. "Je le confesse, je l'avoue ». Mais le maître spirituel de Port-Royal, l'abbé de Saint-Cyran, de qui Pascal a lu les Lettres chrétiennes et spirituelles lors de sa première conversion, en 1646, Saint-Cyran a une conception singulièrement vive et singulièrement large de la vocation. Il ne conseille pas toujours à ses dirigés d'abandonner le monde, loin de là. Le nouveau converti se montrera plus attentif que jamais à bien faire son métier, à remplir ses devoirs dans le monde, car il est là où Dieu a voulu le placer. Idée qui se trouve déjà chez François de Sales, et que nous retrouverons par exemple chez Bourdaloue.

Mais comment concilier cela avec cet « oubli du monde et de tout hormis Dieu » de Pascal et avec la tradition chrétienne, avec l'enseignement de Saint-Jean dans l'Épitre que Pascal cite si souvent : "N'aimez ni le monde ni rien de ce qui est dans le monde »? Avant de répondre à cette objection, constatons d'abord que la Recherche du temps perdu exprime une attitude vis-à-vis du monde et du divertissement qu'on pourrait qualifier de " portroyaliste ", et qui certainement peut être transposée en termes traditionnellement chrétiens. Le narrateur va dans le monde, vit pour le monde. Mais il connaît, - à intervalles toujours plus rares à mesure qu'il se laisse saisir par le monde, - des moments mystérieux, le fameux phénomène de la mémoire involontaire, où il sent chaque fois un appel, - c'est là l'idée même de la vocation, - à approfondir le mystère du phénomène dans la solitude, mais où il s'y soustrait, par paresse, par frivolité, non sans se rendre compte qu'il a failli à son devoir. Ne pourrait-on appeler ces moments privilégiés les appels de la grâce ? « Mais ma grâce est insidieuse, dirait le Dieu de Péguy, et ceux qu'elle veut avoir, elle les a ». Le narrateur peut bien se soustraire à son appel, cela n'empêche que le monde, de son côté, perd pour lui son attraction, se montre de mieux en mieux sous ses vraies couleurs, vanité, égoïsme, cruauté, frivolité. En même temps le narrateur exprime une attitude de plus en plus sévère vis-à-vis de toutes les valeurs, même les plus 
hautes qui ont cours dans le monde. Qui de nous n'a été choqué, du moins à notre première lecture de la Recherche du temps perdu, par les paroles cruelles du narrateur au sujet, non seulement de l'amour, mais de l'amitié ? Et cela même quand il parle de son amitié avec Saint-Loup, qui nous semble en tout point une valeur positive? C'est que le narrateur a une conception du divertissement qui ressemble d'une manière frappante à la conception pascalienne telle que nous la trouvons exprimée dans la huitième liasse titrée, Divertissement. Pascal n'y considère-t-il pas toute activité humaine comme du divertissement, non seulement le jeu, la danse, la chasse et toutes les occupations ordinaires de l'honnête homme ou de ce que nous appellerions le mondain, - mais il appelle divertissement et les condamne comme telles des occupations beaucoup plus sérieuses, comme par exemple «l'apprentissage des langues ${ }^{36}$ ». De même Proust, à bien des reprises, et une dernière fois dans le Temps retrouvé, parle de " cette fuite loin de notre propre vie que nous n'avons pas le courage de regarder, et qui s'appelle l'érudition ».

Une ressemblance aussi frappante pourrait-elle ne pas avoir une cause semblable? Pour Pascal l'apologiste, le divertissement chez l'incroyant est le grand obstacle à sa conversion, parce qu'il l'empêche de penser à ce qu'il est, d'où il vient et où il va. Ainsi « tout le malheur des hommes vient d'une seule chose, qui est de ne savoir pas demeurer en repos dans une chambre ${ }^{37}$ » : c'est la phrase que Proust cite dans sa lettre à Reynaldo Hahn. Pour le narrateur, toute activité qui l'empêche de suivre sa vocation, tout est du temps perdu, même l'amitié, même l'érudition. "Il y a chez Proust une morale de la solitude ", a dit un critique. Je le crois bien. Écoutons le narrateur du Temps retrouvé:

...les vrais livres doivent être les enfants non du grand jour et de la causerie, mais de l'obscurité et du silence.

De même, Proust écrit à son ami Sidney Schiff :

Dire que quand on voit une personne, lire un livre d'elle est inutile... est absurde. Entre ce qu'une personne dit, et ce qu'elle extrait par la méditation des profondeurs où l'Esprit nu gît, couvert de voiles, il y a un monde.

(Et Proust écrit Esprit, avec une majuscule).

Mais j'en viens à une dernière ressemblance entre Proust et Pascal, et cette fois-ci il ne s'agit plus de rapprochement entre l'apologiste ennemi du divertissement, et le narrateur ennemi du monde, mais entre la vie de Pascal et celle de Proust. Les biographes de Pascal ont trop longtemps parlé d'une période mondaine qui se termine par la conversion de la nuit du 23 au 24 novembre, après quoi «oubli du monde et de tout hormis Dieu». Les premiers biographes de Proust ont trop parlé de son snobisme et de son amour du monde, et dans sa vie aussi. On peut voir une sorte de conversion dans le sens de changement de direction, qui commence à la mort de sa mère, s'aggrave par la maladie, et le détourne aussi du monde. Il me semble bien que dans les deux cas nous avons affaire à une légende dont nous sommes en train de nous défaire. La période mondaine de Pascal ? C'est à cette époque qu'il se procure ce Montaigne dans l'édition de 1652 qu'il étudie avec tant de soin... pourquoi ? Sinon pour mieux comprendre ses amis libertins, les mondains de qui Montaigne était la Bible. C'est ainsi qu'au lendemain même de sa conversion, il sera tout armé pour son entreprise de les convertir, comme le montre l'Entretien avec M. de Sacy. «Oubli du monde » après novembre 1654 ? Bien loin de l'oublier, bien loin de rester en repos dans une chambre, Pascal se livrera à ses intenses activités polémiques, apologétiques, pratiques, scientifiques.

87 Et Proust? Sa période mondaine se passera à observer les mondains qui formeront une partie si importante de son œuvre. Son amie Jeanne Pouquet l'avait bien compris, elle qui écrit de Proust à l'époque où, comme elle dit, il se consacre aux Guermantes : 
Car il fut pris pour «le monde » d'une passion incroyable où beaucoup ne virent qu'un snobisme dévorant et qui n'était que l'impérieuse et secrète nécessité de connaître les personnages de son œuvre et le cadre dans lequel il voulait les placer. imaginer contraste plus frappant entre deux styles. Chez Pascal, l'image-éclair, l'antithèse dramatique, la tension continue. Chez Péguy, les litanies interminables, les répétitions et les reprises, les images qui se transforment imperceptiblement au cours de longues pages. Les rapprochements ne font que souligner l'écart. L'exclamation de Pascal :

Qu'il est beau de voir, par les yeux de la foi, Darius et Cyrus, Alexandre, les Romains, Pompée et Hérode, agir, sans le savoir, pour la gloire de l'Évangile ${ }^{38}$ !

Et une fois qu'il sera «converti » à sa vocation d'écrire la Recherche du temps perdu, que voyons-nous? Proust continue à sortir aussi souvent que sa santé le lui permet, et surtout il continue à cultiver ses nombreux amis par sa correspondance. Et c'est dans une lettre à Sidney Schiff que nous trouvons enfin la réponse à notre question : comment être à la fois dans le monde et hors du monde?

Vous avez raison... les conversations sérieuses sont faites pour les gens qui n'ont pas de vie spirituelle. Les gens qui ont une vie spirituelle comme nous trois ont au contraire besoin, quand ils sortent d'eux-mêmes et du dur labeur intérieur, d'une vie frivole.. peut-être chez l'apologiste, et certainement chez le savant qui organise le concours de la roulette, - danger de l'éparpillement chez l'observateur des Guermantes. La maladie s'est chargée de les rappeler à leur vocation. Selon la belle expression de Charles du Bos, ils ont été des «malades par vocation », ou selon celle de Claudel, des « invités à l'attention ». me semble démontrer, c'est qu'il existe aussi des « lois spirituelles », et j'aurais pu donner à cet essai le titre: Proust et Pascal, ou De la vie intérieure.

\section{Péguy et Pascal}

Des trois écrivains que je me suis proposé de considérer dans leur dialogue avec Pascal - Proust, Péguy et Valéry - c'est sans hésitation possible Péguy qui a les affinités les plus profondes avec l'auteur des Pensées, et sur qui l'auteur des Pensées a eu la plus grande influence.

critique. Quand, au lendemain de la dernière guerre, se fondent les Cahiers de l'amitié Charles Péguy, le premier numéro (1947) contiendra une belle étude de Jules Riby, le camarade de Péguy au lycée d'Orléans et à Sainte-Barbe, "Péguy et Pascal. Notes et souvenirs ». Mais ces notes, déjà incomplètes quand elles ont paru, le sont encore davantage aujourd'hui que nous possédons tant de textes inédits de Péguy que Riby n'a pu utiliser, et surtout ceux qu'on appelle les cinq grands inédits, parus dans les années cinquante, L'Esprit de système, Par ce demi-clair matin, Un Poète l'a dit, La Thèse, Clio I, qui nous révèlent clairement l'influence de Pascal.

À première vue, cela peut surprendre : car si le style est de l'homme même, on ne saurait$$
\text { Cest bien l'idée que reprendra Péguy dans son Ève, au huitième clima }
$$

C'est bien l'idée que reprendra Péguy dans son Ève, au huitième climat, qu'il intitule « la légation du monde antique à Jésus par les soins de Rome ", pour la développer sur deux litanies alternantes, en plus de cent alexandrins : 
Il allait hériter des lourds légionnaires...

Et les pas d'Alexandre avaient marché pour lui...

deux à la fois, et remplissant tout l'entre-deux ${ }^{39}$.

$O$ mère ensevelie hors du premier jardin

C'est Jésus qui parle à son aïeule, Ève. contribution, mais Clio l'en empêche : autre fois...

Mais par ailleurs voyons ce que Péguy et Pascal ont en commun, en citant le fragment auquel Proust faisait allusion dans sa lettre à Montesquiou :

On ne montre pas sa grandeur pour être à une extrémité mais bien en touchant les

Pascal et Péguy ont tous deux montré leur grandeur de cette manière-là. Tous deux ont été de féroces pamphlétaires, tous deux sont des poètes mystiques. L'auteur de L'Argent et d'Un nouveau théologien, M. Fernand Laudet, doit être comparé à l'auteur des Provinciales, et le poète des Cinq prières dans la cathédrale de Chartres à celui du Mystère de Jésus. L'un et l'autre remplit « tout l'entre-deux » bien que de manière fort différente : là où Pascal est savant, Péguy est métaphysicien, et au christianisme scripturaire, augustinien, pessimiste de Pascal correspond le christianisme médiéval et optimiste de Péguy.

«Tout pamphlétaire est un homme de dialogue », dit un ouvrage de Péguy pamphlétaire : voilà la deuxième grande ressemblance entre Péguy et Pascal. Pascal, nous l'avons vu, est toujours et partout homme de dialogue, et non pas seulement quand il est pamphlétaire. On pourrait dire la même chose de Péguy. Ses premiers ouvrages ne sont-ils pas, Marcel, dialogue de la cité harmonieuse, qui n'a rien de polémique, et l'énorme drame de la première Jeanne d'Arc (1897)? Toujours Péguy fait parler quelqu'un, ou parle lui-même en s'adressant à quelqu'un. Les grandes œuvres en prose les plus sereines ne sont-elles pas toutes des dialogues: les deux Clio qui ont comme sous-titres: Dialogue de l'histoire et de l'âme païenne, Dialogue de l'histoire et de l'âme charnelle? Victor-Marie, comte Hugo ne s'adresse-t-il pas d'un bout à l'autre à Daniel Halévy? Et la Note conjointe sur M. Descartes ne s'arrête-t-il pas au milieu d'un dialogue entre Péguy et un prêtre?

Il en va de même des œuvres poétiques. Les trois Mystères sont un dialogue, au début entre Jeanne et Hauviette, ensuite entre Jeanne et Madame Gervaise. Les tapisseries invoquent sainte Geneviève ou Notre Dame. Et l'énorme dernier poème, Ève, a comme indication au début du texte : Jésus parle, et comme premier vers:

Évidemment que pour Péguy, encore plus que pour Pascal, il faut prendre dialogue dans un sens large. Les dialogues de Péguy tendent généralement au monologue. Marcel n'est pas un vrai dialogue; si Victor-Marie, comte Hugo a bien le ton d'une conversation entre deux amis, Daniel Halévy est bien obligé de rester muet. $\mathrm{M}^{\mathrm{me}}$ Gervaise, dans le Mystère de la charité de Jeanne d'Arc, laisse encore parler Jeanne d'Arc, mais elle parlera seule tout au long du Porche du mystère de la deuxième vertu. Et la vieille Clio, bien qu'elle ne nous laisse jamais oublier que Péguy est présent et l'écoute respectueusement, ne le laissera parler qu'une fois, et prononcer une seule phrase, au milieu d'un « dialogue » de près de trois cents pages. Quelque cent pages plus loin, il a dû essayer d'y aller avec sa petite

Moins heureux que Barrès [Clio vient de rappeler comment Barrès avait eu la chance de voir en même temps Hugo et Leconte de Lisle à la bibliothèque du Sénat] vous ne reçûtes que le coup de tomber sur Leconte de Lisle, débouchant inopiné du coin sud-est sous les galeries de l'Odéon. Non, non, vous nous conterez cela une

L'œuvre de Péguy est ainsi pleine de personnages qui monologuent pendant des centaines de pages, quand il ne le fait pas en son propre nom. À lire Péguy comme à lire Pascal, on 
entend toujours une voix, et malgré le contraste entre deux styles, Pascal a exercé une influence formatrice sur l'art de Péguy, de même que sur sa pensée et sur l'homme tout entier.

Pascal, rappelons-le, aime le réel et le concret. Les Pensées ne sont pas les matériaux d'une apologie systématique de la religion chrétienne, ce sont les matériaux d'une entreprise pratique, celle de convertir, et tout d'abord ce libertin honnête homme, disciple de Montaigne, que représentent si bien Méré et Mitton. C'est à cause de Pascal que la critique et l'érudition se sont occupées de Méré et de Mitton et qu'elles ont découvert que dans l'un et l'autre cas, la vie du joueur indifférent et sceptique s'est terminée par une vieillesse dévote. S'agit-il de conversions dues à Pascal ? La critique ne se pose pas la question, et elle a sans doute raison, puisqu'il est impossible d'y répondre. Mais admettons que Pascal ait fait des conversions posthumes; il est vraisemblable qu'autour de 1900 il s'est fait un nouveau converti, qui s'appelle Charles Péguy.

Si nous voyons dans la conversion de Péguy l'événement principal de sa vie, qui fait d'un socialiste militant un catholique mystique - et du reste anticlérical et resté en dehors de l'Église visible pour des raisons qui lui font honneur - cette conversion devient publique en 1910 par la publication du Mystère de la charité de Jeanne d'Arc. Mais dès 1908 il avait confié à son fidèle Lotte : «j'ai retrouvé la foi, je suis catholique » et depuis la publication des Mémoires de Raïssa Maritain, nous savons qu'en janvier 1907 il avait fait la même confidence à Jacques Maritain. Et il faut remonter sans doute encore plus haut, car nous n'avons pas affaire à une conversion dramatique, spectaculaire, comme celle de Paul Claudel, adossé à un pilier de Notre-Dame de Paris, le 25 décembre 1886, à Vêpres, au chant du Magnificat. Il serait impossible de placer une plaque commémorative de la conversion de Péguy, mais ce qui est certain, c'est que Pascal a été le compagnon fidèle du long itinéraire.

104 Jules Riby témoigne que Péguy fut un auditeur assidu, en 1897, du cours professé en Sorbonne sur Pascal par Émile Boutroux :

Un jour notre maître se posa, entre mille autres, cette question: Pascal est-il socialiste? Question passionnante, pour le jeune auditoire d'admirateurs de Jaurès que nous étions alors. Et après avoir cité les textes fameux : l'égalité des biens est juste , et puis, ce chien est à moi... et l'usurpation de toute la terre, toujours de la même voix douce et un peu voilée, il nous donna lecture en guise de conclusion, de ces lignes de $\mathrm{M}^{\mathrm{me}}$ Périer : « Tous ces discours nous excitaient et nous portaient quelquefois à faire des propositions pour trouver des moyens pour des règlements généraux qui pourvussent à toutes les nécessités; mais il [Pascal, son frère] ne trouvait pas cela bon et disait que nous n'étions pas appelés au général mais au particulier et qu'il croyait que la manière la plus agréable à Dieu était de servir les pauvres pauvrement, c'est-à-dire chacun selon son pouvoir, sans se remplir l'esprit de ces grands desseins qui tiennent de cette excellence dont il blâmait la recherche en toutes choses. » Je n'ai pas oublié, étant ce jour-là le voisin immédiat de Péguy, la vive impression que fit sur lui cette lecture et l'étonnement mêlé de quelque impatience qu'il marqua en l'écoutant...

Riby commente que le choc, l'ébranlement que reçut ce jour-là le jeune socialiste Péguy fut le point de départ d'une évolution qui devait le conduire à proclamer : «la révolution sera morale ou ne sera pas !», qui allait devenir la devise des Cahiers de la quinzaine. Mais il faut sans doute remonter encore plus haut, jusqu'à l'année 1889-1890, où, au lycée d'Orléans, Péguy a fait sa rhétorique et lu les Pensées dans l'édition Havet. L'édition classique de Havet, c'est son Pascal, et il l'est resté toute sa vie, comme il nous le dit à maintes reprises dans ses œuvres. Péguy qui s'est tant moqué de la «méthode scientifique " en littérature, ne le cède en rien aux Lanson pour les citations et les 
références. Il citera surtout les notes de « $\mathrm{M}$. Havet » avec une malice d'où l'affectation n'est pas absente. Il dira en 1905 : «Je copie ce Pascal dans ma vieille petite édition Havet ; je ne sais ce que j'ai fait de mon Brunschvicg ${ }^{40}$. »

Quand il parle de Havet, neuf fois sur dix, il s'agit de l'édition des Pensées, mais il mentionne au moins une fois l'édition Havet des Provinciales, ce qui ne nous surprend pas, car on peut bien dire que Péguy entre dans la littérature sous le signe des Provinciales. Rappelons comment.

En décembre 1899 a lieu le Congrès socialiste, d'où Péguy sort écœurer et qui mène à la fondation des Cahiers de la quinzaine, ces Cahiers dont il sera le gérant jusqu'à sa mort, et où paraitront toutes ses œuvres.

Le 5 janvier 1900 paraît le premier Cahier. On s'attendrait à y trouver un Avis de l'éditeur qui donnerait le programme de la nouvelle revue, qui indiquerait ses principes et sa méthode; et c'est bien ce que nous donne Péguy, mais d'une manière inattendue. Il adopte la fiction de Pascal, en renversant l'ordre des rôles : au lieu de commencer par une lettre de Louis de Montalte à un Provincial de ses amis, nous trouvons la Lettre du Provincial, datée, « de la Province, jeudi 21 décembre 1899 », commençant : « Mon cher Péguy ». C'est à ce professeur de l'enseignement secondaire, ardent dreyfusiste, que Péguy confère le rôle de critiquer le congrès socialiste, ce congrès qui avait voulu supprimer la liberté de la presse socialiste, et il le fait en socialiste naïf qui a toujours cru que le premier devoir était «l'obligation de dire la vérité ». Cela le conduit à prier Péguy de lui envoyer toutes les quinzaines un cahier de renseignements et à lui indiquer le programme à remplir : « Tu me diras ce que tu verras... Tu me diras ce que tu penses... Tu me transcriras tous les documents et tous les renseignements qui sont à conserver... ", et ainsi de suite. Et Péguy d'intercaler malicieusement une phrase qui révèle bien son modèle littéraire et qui correspond à la courte réponse du Provincial à Pascal :

$\mathrm{Tu}$ iras voir les docteurs que tu connais, et tu leur demanderas pour moi des consultations sur les cas difficiles.

Péguy publie ensuite une courte Réponse datée de Paris, où il accepte la proposition du Provincial. Suit alors immédiatement le premier reportage : Le Triomphe de la République. Mais le mois suivant, février 1900, Péguy tombe malade de la grippe, alors qu'il avait l'intention d'aller voir « le docteur moraliste révolutionnaire ». La grippe était bien réelle, mais le citoyen docteur est sans doute un personnage fictif. Heureusement que ce citoyen docteur vient le voir, et nous avons les brillants cahiers De la Grippe, Encore de la grippe, Toujours de la grippe, composés tout entiers de dialogues où l'imitation des Provinciales est évidente : quand les deux amis discutent de la révolution sociale, ou du parti socialiste, le dialogue de Péguy offre le même mélange de naïveté et d'ironie que le dialogue pascalien. Mais c'est dans ces mêmes textes que Péguy introduit «L'autre Pascal », non le maître littéraire, mais le chrétien qui travaille à le convertir, pendant sa maladie, dit-il au citoyen docteur, il a pris sa petite édition classique des Pensées de Pascal, et

...dans cette édition qui me rappelait de bons souvenirs, je lus la Prière pour demander à Dieu le bon usage des maladies [éd. Havet, p. 553-565]. J'admire comme on le doit cette passion religieuse et, pour dire le mot, cette foi passionnément géométrique, géométriquement passionnée...

110 La phrase continue pendant quatre lignes encore, pour s'achever brusquement sur étrangère à nous, à quoi le docteur répond :

Moins étrangère que vous ne le croyez. 
111 La réponse nous montre que ces dialogues de Péguy sont de véritables dialogues entre deux aspects de sa pensée et de sa personnalité. Il s'exprime à la fois dans ses deux personnages, le «moi » et le docteur fictif. Le malade continue son récit par cet aveu significatif :

J'étais heureux de lire du Pascal, parce que j'ai gardé pour ce chrétien une admiration singulière inquiète.

112 Dans le Cahier suivant, Encore de la grippe, ils reviendront à Pascal, et le docteur demandera à Péguy de lui passer sa petite édition des Pensées pour lui faire lecture de longs passages de la Vie de Blaise Pascal par Madame Périer (Gilberte Pascal) [ibid.]. Les dernières pages de cette Vie, sur la dernière maladie et la mort de Pascal, sont transcrites tout au long, et le Cahier se termine sur ce court paragraphe :

Quand le docteur eut fini de me lire tout ce qu'il avait librement choisi dans l'histoire de la vie et de la mort de Blaise Pascal, je ne pensai pas à lui demander pourquoi il m'avait fait une aussi longue citation; mais nous demeurâmes longtemps sous l'impression de ce témoignage ${ }^{41}$.

113 Toujours de la grippe reprend le thème de Pascal, mêlé à beaucoup d'autres, et cite, cette fois, les Pensées : le fragment des trois ordres tout entier ${ }^{42}$; et celui du roseau pensant ${ }^{43}$; avec commentaires. Mais brusquement, dans les dernières pages, Péguy exprime son horreur de la foi chrétienne, à cause de la doctrine de la mort éternelle. Nous savons que cette obsession de l'enfer, il la donnera encore à sa Jeanne dix ans plus tard, dans le Mystère de la charité de Jeanne d'Arc, et c'est alors $\mathrm{M}^{\text {me }}$ Gervaise qui lui donne la réplique. Le dialogue à l'intérieur de Péguy continuera presque jusqu'à la fin de sa vie.

114 Tout de même, cette présence de Pascal dans trois textes de la première série d'une publication qui s'était annoncée comme politique et socialiste, n'est-elle pas extraordinaire ? Et Péguy va récidiver. Dans la troisième série, il dira : Nous devons nous préparer aux élections, et après avoir constaté avec tristesse, et exprimé son horreur de ce qu'il appelle « la prostitution électorale », il pose la question : "Tout cela n'est-il qu'un immense divertissement?» Suivent alors immédiatement, sans autre explication, sept pages en italiques ${ }^{44}$ : ce sont les fragments pascaliens sur le Divertissement, qui forment dans l'édition Havet les trois premiers fragments de l'article IV, en tête le long texte: "Quand je m'y suis mis quelquefois, à considérer les diverses agitations des hommes... ", Au moment où Pascal va dire : Le roi est environné de gens qui ne pensent qu'à divertir le roi, et l'empêcher de penser à lui. Car il est malheureux, tout roi qu'il est, s'il y pense ", Péguy intercale :

Ces vieilles paroles reçoivent une extension prodigieuse, aujourd'hui que tant de penseurs ont annoncé, que tant d'ouvriers ont préparé, que tant de soldats ont installé, que tant de poètes ont chanté, que tant d'historiens ont conté la démocratie, le peuple souverain, le peuple roi.

Le dernier commentaire suit la fin du fragment sur la dignité royale : «Je ne parle point en tout cela des rois chrétiens comme chrétiens, mais seulement comme rois. » et Péguy d'ajouter : «Qu'on remplace roi chrétien par peuple républicain ${ }^{45}$. »

116 Ailleurs dans ces premiers cahiers, Péguy reprend le ton et le dialogue des Provinciales, avec une drôlerie irrésistible. Entre deux trains met en scène René Lardenois, qui vient voir le gérant des Cahiers; Péguy lui répète ce qu'a dit «notre ami Baudouin » de ses premiers cahiers. Il lui a reproché de s'attarder à commenter les événements, ce qui est malsain, et d'avoir eu peur de se rendre ridicule en traitant des grandes questions, comme de l'immortalité de l'âme : 
Pressé de toutes ces peurs, tu nous as donné quelques misérables citations du grand Pascal, citations lamentablement mesquines et déplorablement tronquées et inconvenablement brèves... 
même l'esprit de Pascal, c'est qu'un de ces ordres correspond souvent à l'ordre pascalien de la charité. Ainsi nous avons les deux couples temporel / éternel, ou charnel / spirituel. $\mathrm{Et}$, comme chez Pascal, il s'agit de voir où, dans la nature humaine, est le point d'insertion de la grâce - et ici il faut revenir à la notion du cœur chez Pascal. On a pu résumer toute la pensée et toute l'œuvre de Péguy comme centrée sur la doctrine de l'incarnation, qui est le point où l'éternel entre dans le temporel, où le spirituel s'insère dans le charnel. La notion d'ordre, au sens de catégorie distincte, se trouve aussi appliquée dans des contextes tout différents, par exemple l'ordre de l'action et l'ordre de la connaissance. Ou encore, Péguy développe l'idée que le talent et le génie appartiennent à deux ordres distincts :

... et j'irai jusqu'à dire cette loi, ... que le génie n'est point du talent porté à un très haut degré, ni même du talent porté au plus haut degré, ni même à sa limite, mais qu'il est d'un autre ordre ${ }^{48}$.

Dans ce passage on devine bien, comme sous-jacents, deux grands textes de Pascal, celui du fragment S. 339 / L. 308 et aussi celui de la première page du fragment Infini-rien ${ }^{49}$ : «L'unité jointe à l'infini ne l'augmente de rien... ».

Ceci nous conduit à la notion du "pari » chez Péguy, qui se retrouve aussi constamment dans son œuvre que dans sa vie. Halévy l'a bien compris, et il faut citer ce beau passage de son beau livre:

Chaque âme a son signe distinctif, qui marque sa vie, sa sainteté, si elle est sainte. Pour l'une, c'est la charité : elle veut toujours donner; pour telle autre, c'est pauvreté : elle ne se sent jamais assez dénuée. La marque de l'âme de Péguy, son génie, c'est le risque : il ne se croit jamais assez exposé ${ }^{50}$.

Est-ce pour cette raison que Péguy a si bien compris le «pari » de Pascal ? Dans notre premier chapitre, nous avons dit que Pascal insiste sur la nécessité du pari, sur l'inévitable nécessité de choisir, et aussi que tout choix comporte un risque, et nous avons tenté de montrer que cette idée a des applications presque infinies. Chez le jeune Proust, nous avons trouvé un texte qui rappelait singulièrement la glose de Port-Royal : « ...et ne point parier que Dieu est, c'est parier que Dieu n'est pas. » Dans une des innombrables pages où Péguy attaque ce qu'il appelle «le parti intellectuel moderne », et qui est sans doute l'aspect qu'il hait le plus de ce "monde moderne » auquel il a déclaré la guerre, dans le cahier sans titre du 3 février 1907 se trouve ce passage :

Rien n'est aussi difficile que de faire comprendre à celui qui ne le veut pas qu'on a beau nier, qu'on fait tout de même de la métaphysique, et tout de même de la philosophie, et tout de même de la religion, - que généralement ne pas prendre position, ne pas occuper certaines situations, c'est infailliblement en prendre et en occuper d'autres ${ }^{51}$.

Et, deux ans plus tôt, dans le Cahier La délation aux droits de l'homme, Péguy attaquait déjà l'historien moderne avec sa folle prétention d'examiner tous les documents, ce qui le conduisait à déclarer :

... car s'il ne veut pas choisir, dans son fol orgueil de savant moderne, s'il refuse formellement de choisir, il choisit quand même, car il choisit l'écrasement, l'inexistence, et la stérilité ${ }^{2}$.

Cela le conduit à deux belles pages de retour sur lui-même, à cette "effrayante responsabilité de choisir qu'il a assumée... » et qui illustre bien le commentaire de Halévy que nous avons cité. Mais cela ne nous rappelle-t-il aussi la citation de Sartre (commentée ci-dessus, chap. 1) : «Je peux toujours choisir, mais je dois savoir que si je ne choisis pas, je choisis encore »? 

savait encore ni lire ni écrire. libertin. et tout doucement cet $I l$ se transforme en Pascal. mélancolie. conversion.

\section{Valéry et Pascal}

Il me semble bien que Péguy a donné la formule de l'existentialisme quand Sartre ne

Notre dernier point de comparaison sera ce que nous avons appelé dans notre premier chapitre «la prétendue angoisse de Pascal», malentendu qui repose sur cinq ou six fragments des Pensées à la première personne, et qu'il faut mettre dans la bouche du

Péguy ne se prononce jamais, que je sache, sur ce problème du : qui parle ? Il semblerait plutôt adopter l'attitude ambiguë des commentaires de Havet qui, après avoir noté, à propos du monologue de l'athée, que c'est le libertin qui parle, dit ensuite simplement $I l$

Je n'accuse pas Péguy d'ambiguïté. Plus simplement, il dit Pascal en parlant de l'écrivain qui a su admirablement exprimer la misère de l'homme sans Dieu. Jules Riby marque fort bien l'importance du thème "grandeur / misère " chez Péguy. Mais Péguy ne semble guère avoir médité sur le "silence éternel ». Quand il parle de la " royale détresse » d'un Pascal, il fait allusion aux « misères d'un roi dépossédé ", des fragments S. 148, 149/ L. 116 et 117 de la liasse Grandeur $^{53}$. Il en parlera avec magnificence dans la dernière de ses œuvres en prose, que beaucoup considèrent comme son chef d'œuvre, la Note conjointe, inachevée. Il y parlera de l'inquiétude juive greffée dans le corps chrétien.

Il fallait cela (aussi il fallait doublement cela) pour que fût produit un Pascal, pour que fussent obtenus ce puits de détresse, ce désert de sable, cet abîme de

Comment combattre un malentendu qui a donné naissance à une phrase aussi belle ? Une phrase qui, par le rythme et les images, par ce que Claudel appelle «le ballon de l'incidence », est digne de figurer à côté de la phrase pascalienne du silence éternel ?

Ce qui est certain, c'est que Péguy a connu l'inquiétude, c'est de lui-même aussi qu'il parle. Et nul ne niera que Pascal l'apologiste donne à l'inquiétude aussi une valeur positive, puisque c'est précisément cette inquiétude qu'il cherche à créer chez son libertin, afin qu'il devienne celui qui « cherche en gémissant ».

Et si, de la dernière œuvre en prose de Péguy, nous passons à sa dernière œuvre poétique, l'immense poème Ève, et que nous le lisons avec cet excellent guide qu'est Albert Béguin, que verrons-nous? À son "essai de lecture commenté » Béguin donne des sous-titres pascaliens : le divertissement, l'ordre de la charité, ni ange ni bête...

Concluons : le Pascal des Provinciales a appris à Péguy son métier de polémiste. Le Pascal des Pensées a nourri la pensée métaphysique et religieuse de Péguy et a dû contribuer à sa

Si le dialogue de Péguy avec Pascal est celui d'un disciple avec son maître, celui de Valéry avec Pascal a lieu dans un climat tout différent, que nous pourrions caractériser en comparant les lettres de Voltaire où il parle de son «petit anti-Pascal ", et la lettre de Valéry à son ami Pierre Louÿs du 21 mai 1917, où il lui annonce le projet d'un « éreintement sauvage » de Pascal ${ }^{54}$. Le ton est différent, l'intention est semblable.

Maurice Bémol appelle Pascal «l'ennemi intime » de Valéry. Et, ajouterons-nous, celui-ci est d'autant mieux équipé pour cette guerre qu'il est lui-même homme de dialogue. Un 
ouvrage sur Valéry a comme sous-titre : A study in dramatic monologue - ouvrage de Francis Scarfe, The art of Paul Valéry. L'auteur y considère toute l'œuvre du poète sous cet angle du monologue dramatique, qui le conduira, par une remarquable évolution, à écrire des dialogues, puis des libretti, enfin une vraie pièce pour la scène : Mon Faust. 
Voici d'abord, à la fin d'Analecta (1926), le fragment S. 455 / L. 530 réfuté. Pascal a écrit : « Tout notre raisonnement se réduit à céder au sentiment ». Valéry commente :

Si tout notre raisonnement se réduit à céder au sentiment, c'est celui qui cède qu'il faut plaindre... Mais ce n'est pas le raisonnement qui cède. C'est moi. - Qui, Moi ? Celui qui agit. Car l'autre est variation illimitée; il reviendra sur son sentiment; il se reprendra au raisonnement. Et ainsi de suite...

Valéry a ajouté une note à sa citation de Pascal : «C'est là une idée de Pythie, l'idole de l'oracle. Le spontané, l'irréfléchi plus précieux, plus digne de foi, que le réfléchi ${ }^{56}$. »

Voilà donc Pascal taxé d'avoir une «idée de Pythie ». Mais la suite du fragment S. 455/ L. 530 montre bien que Pascal prend ici sentiment au sens d'intuition, par opposition à la fantaisie :

Mais la fantaisie est semblable et contraire au sentiment, de sorte qu'on ne peut distinguer ente ces contraires. L'un dit que mon sentiment est fantaisie, l'autre que sa fantaisie est sentiment. Il faudrait avoir une règle. La raison s'offre, mais elle est ployable en tous sens; et ainsi il n'y en a point.

Le commentaire de Havet ne peut avoir influencé Valéry dans son interprétation, car il met en note au mot de fantaisie : «C'est la sensibilité variable de chacun » (note à VII. 4).

Dans les Mauvaises pensées, qui sont de 1941-42, Valéry note :

Les méditations sur la mort (genre Pascal) sont le fait d'hommes qui n'ont pas à lutter pour leur vie, à gagner leur pain, à soutenir des enfants ${ }^{57}$.

Remarque fort juste, que Montaigne avait exprimée tout aussi bien. Mais quel mépris dans la petite parenthèse : " genre Pascal »! Et Valéry continue :

L'éternité occupe ceux qui ont du temps à perdre. Elle est une forme de loisir.

Et cette idée de Valéry se retrouve ailleurs, par exemple dans Robinson, Histoires brisées ${ }^{58}$.

Dans Moralités, qui est de 1941, Valéry note brièvement :

Le moi est haïssable ... mais il s'agit de celui des autres ${ }^{59}$.

Onze ans plus tôt, il avait écrit sur cette pensée de Pascal ${ }^{60}$ un développement beaucoup plus sérieux :

Que si le moi est haïssable, aimer son prochain comme soi-même devient une atroce ironie $^{61}$.

57 Ici Valéry, avec sa logique impitoyable et absurde rappelle cette confidence de Péguy à Lotte: «Pascal raisonne trop, alors les incroyants lui font des colles. » L'intention de Valéry est visible, il veut faire une colle à Pascal. Ce fragment S. 494/ L. 597, qui a la forme d'un dialogue avec Mitton, s'adresse au non-converti honnête homme. La réponse de Pascal à Valéry, il faut la chercher dans la liasse Morale chrétienne.

Nous avons vu comment Proust et Péguy ont tous deux très bien compris la notion pascalienne du " pari », et cette nécessité où nous sommes de faire un choix. Valéry aussi a fort bien compris, voyez plutôt cette Mauvaise pensée ${ }^{62}$ :

L'homme inconsciemment parie presque tout le temps de sa vie qu'il ne sera pas foudroyé dans les dix minutes ou dix secondes suivantes...

Mais voici comment Valéry poursuit :

" Il a le sentiment de sa propre continuité, et ceci nécessairement »

Et nous sentons pointer la réfutation de Pascal. L'homme pour Valéry est nécessairement dans cet état de "nonchalance " qui est un monstre pour Pascal, et cela est encore souligné par le titre que Valéry donne à sa pensée : L'Espoir fonctionnel, normal. 
On pourrait multiplier ces réfutations de Pascal. Mais deux textes des Pensées par-dessus tous les autres ont été l'objet renouvelé des attaques impitoyables de Valéry: c'est d'abord la phrase toujours citée: "Le silence de ces espaces infinis m'effraie ${ }^{63}$ ", et l'autre: "Tu ne me chercherais pas si u ne m'avais trouvé ${ }^{64}$ ", que nous venons de rencontrer à la fin de Degas, danse, dessin, et qui nous est aussi familière : c'est la réponse à la méditation de Pascal dans le Mystère de Jésus. Cette méditation sur le premier des mystères douloureux du rosaire, l'Agonie au Jardin, se termine par : "Jésus étant dans l'agonie et dans les plus grandes peines, prions pour longtemps!", mais le texte continue alors : «Console-toi : tu ne me chercherais pas si tu ne m'avais trouvé ».

Parlons d'abord de ce second texte. Valéry l'a appliqué à toute la pensée de Pascal. Il ne se lasse pas de répéter que Pascal ne cherche pas, parce qu'il croit avoir trouvé. Or, pour Valéry, c'est bien le péché mortel que d'avoir trouvé, de ne plus chercher. Ici, faisons une digression qui n'en est pas vraiment une; c'est encore une anecdote que nous trouvons dans le Journal de Julien Green.

Le 28 janvier 1951, il rend visite à André Gide :

... (Gide) se tourne vers Jean D..., et lui dit : « Nous allons demander à Green où se trouve la phrase dont nous parlions tout à l'heure. » Et me regardant bien en face, d'un air un peu sévère, il me demande : «D'où est tiré : Tu ne me chercherais pas si tu ne m'avais pas déjà trouvé ?» Et j'ajoute plus haut: "Cette phrase est tirée du Mystère de Jésus (J'ai caché de mon mieux ma stupéfaction. Est-ce vraiment Gide qui me pose cette question?) «Ah! s'écrie-t-il, ce n'est donc pas dans l'Évangile. »

164 Arrêtons ici notre lecture de Green. Vous voyez qu'il est doublement choqué : par l'ignorance de Gide, et aussi par la manière dont il estropie la grammaire de la phrase de Pascal, en ajoutant pas et déjà. Erreur en effet surprenante chez un écrivain classique comme Gide - mais erreur que nous trouvons déjà chez Valéry, mort six ans avant cette conversation.

Dans l'Idée fixe (1931), ce dialogue étincelant d'esprit entre Valéry (Moi) et le Docteur, Valéry attaque l'idée que l'homme moderne devient plus profond parce que «les uns croient pénétrer dans les couches primaires de leur existence ... Ils y cherchent généralement des fossiles obscènes. » À quoi le Docteur répond : « Ils ne les chercheraient pas s'ils ne les avaient pas déjà trouvés. » Comme boutade dirigée contre la psychanalyse, c'est spirituel ; mais il est permis de trouver cette parodie du Mystère de Jésus de mauvais goût. Du reste, tout ce dialogue de deux hommes à la mer est farci de parodies de Pascal, et deux pages plus loin, Moi avoue qu'il était agacé : «Profond et profondeur m'exaspéraient », à quoi le Docteur répond :

- Je parie que vous aviez lu quelque article sur Pascal. - Je ne tiens pas ce pari. Pas

plus que celui de Pascal.

Pour en revenir à la phrase de Pascal, il est frappant de voir Gide et Valéry estropier la citation. Cela me fait soupçonner qu'il s'agirait peut-être chez eux d'une certaine " mauvaise volonté ». Je vous rappelle que Mon Faust s'adressera « Au lecteur de bonne foi et de mauvaise volonté ", ce qui évidemment, pour Valéry, est préférable à l'inverse. Et je note que Montherlant dans ses Carnets, après avoir dit que c'est la lecture de Pascal, alors qu'il était adolescent, qui lui a fait perdre la foi, ajoute : « Tu ne me perdrais pas si tu ne m'avais perdue » et respecte ainsi la phrase de Pascal même en la transformant.

Passons au «silence éternel» qui a donné lieu au célèbre texte de Valéry publié à l'occasion du tricentenaire de la naissance de Pascal, en 1923: Variation sur une pensée ( Variété I). Avant de l'analyser, rappelons notre position (supra, chap. 1). Ce qu'on appelle 
communément l'angoisse de Pascal repose sur un grand malentendu, sur une demidouzaine de fragments à la première personne qu'il faut mettre dans la bouche du libertin, et qu'on a trop longtemps pris pour l'expression des sentiments personnels de Pascal. Ces fragments, ce sont surtout notre fragment S. 233 / L. 201, et les fragments S. 227 et 229/ L. 194 et 198 que Pascal a également classés dans la liasse Transition de la connaissance de l'homme à Dieu :

Pourquoi ma connaissance est-elle bornée ${ }^{65} ? \ldots$

En voyant l'aveuglement et la misère de l'homme, en regardant tout l'univers muet

et l'homme sans lumière... et comme égaré dans ce recoin de l'univers ${ }^{66} .$.

Nous avions ensuite cité le «monologue de l'athée » du fragment S. 681 / L. 417 : «Je ne sais qui m'a mis au monde... ». Je voudrais maintenant revenir à ce monologue, car je ne veux pas simplifier le problème, ni passer sous silence une objection qu'on peut faire à notre assertion : c'est le libertin qui parle, et non Pascal. Cette objection, qui est de poids, est celle-ci : jamais un libertin n'a parlé de la sorte, et les Méré et les Mitton n'ont sans doute jamais songé à s'effrayer à la pensée des espaces infinis. Nous en tombons d'accord. Alors, continue l'avocat du diable, alors pourquoi prêter au libertin ces sentiments sinon parce que Pascal lui-même les a éprouvés ? La réponse à cette objection, l'explication de l'énigme, se trouve dans ce monologue de l'athée dont il faut maintenant citer la seconde moitié :

... Je ne vois que des infinités de toutes parts, qui m'enferment dans un atome et comme une ombre qui ne dure qu'un instant sans retour. [Cette dernière image est prise d'un des livres sapientiaux, l'Ecclésiaste VI.12.] Tout ce que je connais est que je dois bientôt mourir ; mais ce que j'ignore le plus est cette mort même que je ne saurais éviter.

Comme je ne sais d'où je viens, aussi je ne sais où je vais; et je sais seulement qu'en sortant de ce monde je tombe pour jamais ou dans le néant ou dans les mains d'un Dieu irrité, sans savoir à laquelle de ces deux conditions je dois être éternellement en partage. Voilà mon état, plein de faiblesse et d'incertitude. Et de tout cela je conclus que je dois donc passer tous les jours de ma vie sans songer à chercher ce qui doit m'arriver. Peut-être que je pourrais trouver quelque éclaircissement dans mes doutes; mais je n'en veux pas prendre la peine, ni faire un pas pour le chercher ; et après, en traitant avec mépris ceux qui se travailleront de ce soin, [...] je veux aller, sans prévoyance et sans crainte, tenter un si grand événement, et me laisser mollement conduire à la mort, dans l'incertitude de l'éternité de la condition future.

Il est clair, n'est-ce pas, que pareil discours n'a jamais été tenu par un libertin en chair et en os. Pascal a composé un amalgame monstrueux en donnant à l'homme qui parle, d'abord des caractéristiques réelles. Nous reconnaissons en lui le disciple de Montaigne; il ne dit pas : « que sais-je ? », mais il répète à satiété : je ne sais, je ne sais. C'est aussi chez Montaigne qu'il a appris à se laisser aller «mollement » à la mort, «sans prévoyance et sans crainte ». Mais en même temps il est satisfait de son ignorance, et il traite avec mépris ceux qui cherchent; ce qui appartient plutôt au type de libertin que nous connaissons par Molière. Il est clair aussi qu'il accepte l'alternative de Pascal, celle du fragment Infini-rien, Dieu est ou il n'est pas, - mais s'il est, c'est le Dieu biblique et le Dieu de Pascal ; c'est pourquoi il admet «qu'en sortant de ce monde je tombe pour jamais ou dans le néant ou dans les mains d'un Dieu irrité ». Mais de plus, Pascal lui fait dire ce qu'il ne dirait jamais, mais qu'il devrait dire : «je suis dans une ignorance terrible » et surtout "Je vois ces effroyables espaces de l'univers qui m'enferment... ». Si le libertin vit, comme il le fait, dans l'hypothèse implicite que Dieu n'existe pas, l'univers ne devient-il pas effrayant, et les espaces infinis muets? Croire à l'harmonie des sphères, n'est-ce pas 
croire implicitement au créateur des sphères ? C'est ainsi que nous nous dirigeons vers le dialogue de Valéry avec Pascal, et que nous revenons à la Variation sur une pensée de 1923 qu'il nous faut maintenant résumer rapidement.

Valéry contraste l'attitude de Pascal avec celle des anciens Grecs qui entendaient la musique des sphères, et celle des Juifs, celle du Psalmiste, pour qui les cieux énoncent la gloire de Dieu :

Pascal ne reçoit des espaces infinis que le silence. Il se dit «effrayé ». Il se plaint amèrement d'être abandonné dans le monde. Il n'y découvre pas Celui qui déclarerait à Jérémie : Coelum et terram ego impleo. Et cet étrange chrétien ne se trouve pas son père dans les cieux... Mais au contraire, « en regardant tout l'univers muet, il entre en effroi, dit-il, comme un homme qu'on aurait transporté endormi dans une île déserte et effroyable ${ }^{67} \ldots$.. .

Effroi, effrayé, effroyable: silence éternel, univers muet, c'est ainsi que parle de ce qui l'entoure l'une des plus fortes intelligences qui aient paru.

Donc, pour Valéry, Pascal est un étrange chrétien. Mais ce n'est pas tout. Valéry découvre dans cette attitude de Pascal du parti pris, une volonté de désespoir, qui le conduit à une seconde accusation :

Je ne puis m'empêcher de penser qu'il y a du système et du travail dans cette attitude parfaitement triste et dans cet absolu de dégoût. Une phrase bien accordée exclut la renonciation totale.

Ici on reconnaît l'allusion à la ligne du Mémorial : « renonciation totale et douce ». Donc, Pascal écrit trop bien pour être sincère, et il faut se garder de confondre l'homme et l'artiste. La critique pascalienne contemporaine de Valéry aurait bien fait d'écouter cet avertissement salutaire. Valéry quitte ensuite Pascal pour méditer à son tour devant le ciel étoilé, - ou plutôt pour faire une admirable analyse de l'effet mystérieux que produit sur l'homme une nuit étoilée, sujet auquel il reviendra ailleurs, ainsi dans Eupalinos qui est de la même année, non sans y mêler une allusion ironique au fragment S. 233 / L. 201. Après avoir fait dire à Phèdre que "[les chiens] aboient inlassablement vers la lune ", Socrate continue : «Et les humains, de mille manières, ne s'efforcent-ils pas de remplir ou de rompre le silence éternel ... qui les effraye ${ }^{68}$ ?».

En résumé, toute la religion de Pascal, et toute sa sincérité, toute son honnêteté intellectuelle, dirions-nous aujourd'hui, sont mises en doute à partir de deux ou trois textes mal interprétés. On reste un peu étonné devant tant de légèreté de la part de Valéry. Il est vrai qu'il dira dans une lettre d'août 1923 : «l'article sur Pascal est besogne courante; rien de digne d'être communiqué à d'autres qu'aux quarante mille lecteurs de la Revue hebdomadaire». Mais sept ans plus tard, le voilà qui récidive et qui enrichit le texte de sa Variation de notes inédites. Après avoir analysé la phrase de Pascal comme un poème, il reprend ses accusations et ne s'en excuse pas :

J'ai vu distinctement l'écrivain et même le poète, et presque le rhéteur, dans les quelques mots du silence éternel... Mais je me suis naïvement demandé si un homme qui ne regarde plus qu'en soi et qu'en Dieu ... a le cœur de songer encore au jeu d'écrire?

Il me semble que, placés dans le contexte de la pensée apologétique de Pascal, ces textes à la première personne réfutent les deux accusations de Valéry. Pascal chrétien, et aussi Pascal écrivain, poète, rhéteur, met tous ses dons, tout son « art de persuader » au service de son entreprise de conversion. L'erreur (si naïve !) de Valéry l'a conduit à être d'accord avec Pascal quand il pense le réfuter, - ce que va éclairer un autre texte de Valéry.

En 1927 paraît Autres rhumbs, ou Tel quel, et nous y trouvons cette note : 
Contre épreuve, négatif, d'une phrase illustre : le vacarme intermittent des petits coins où nous vivons nous rassure.

Je ne sais si Valéry a écrit cela avec un petit sourire ironique, le sourire de celui qui a joué un bon tour à son ennemi. Mais on peut lui répondre : Pascal serait enchanté, il dirait à Valéry-Mitton: "Vous m'avez parfaitement compris, c'est ainsi que se comporte l'incrédule qui se divertit, c'est ainsi que vous vous comportez ». Et peut-être que Valéry serait d'accord.

Il me semble que Valéry se divertit de plus en plus aux dépens de Pascal dans les vingtcinq dernières années de sa vie, car la parodie de Pascal y est très fréquente. Le même $\mathrm{Tel}$ quel contient une autre note, qui est encore une flèche lancée à notre fragment S. 93 / L. 201 :

Toute parole a plusieurs sens dont le plus remarquable est assurément la cause même qui a fait dire cette parole.

Ainsi : Quia nominor leo ne signifie point : Car lion je me nomme, mais bien : Je suis un exemple de grammaire.

Dire : le silence éternel etc., c'est énoncer clairement: Je veux vous épouvanter de ma profondeur et vous émerveiller de mon style.

Mais la parodie de Pascal devient de plus en plus légère, spirituelle, ainsi dans l'Idée fixe ou Deux hommes à la mer, que j'ai déjà cité, dialogue qui est de 1931. On sent Valéry de bonne humeur, et comment, si l'on est sensible au style, ne pas se laisser divertir? Cela est vrai aussi de sa dernière œuvre majeure, Mon Faust, écrit quand Valéry approchait de ses 70 ans. Cette pièce inachevée a quatre personnages principaux : Faust et Méphistophélès, $M$ ${ }^{1 l e}$ Lust, la secrétaire de Faust, et le Disciple, - à quoi il faut ajouter les trois démons du III ${ }^{\mathrm{e}}$ acte. Tous ces diables s'en donnent à cœur joie de parodier non seulement Pascal, mais aussi l'Évangile et la liturgie, en quoi ils sont bien dans leur rôle de démons. Puis, voici la scène entre le Disciple et Méphistophélès. Le Disciple se réveille, raconte son rêve, et conclut : « Non, c'est indescriptible. Je dis des choses absurdes », à quoi Méphisto répond : «L'absurde a ses raisons, Monsieur, que la raison soupçonne ». Méphisto éclaire alors la bibliothèque de Faust, et le Disciple de s'écrier: «Le silence éternel de ces volumes innombrables m'effraie ».

Mais quittons ces parodies gracieuses pour revenir en arrière et poser de nouveau la question: Comment Valéry a-t-il pu si mal comprendre Pascal sur cette question de l'angoisse?

Rappelons d'abord que l'époque ne pouvait qu'encourager ce malentendu. Valéry a fait sa rhétorique au lycée de Montpellier, de 1886 à 1887. Il a lu les Pensées dans l'édition Havet, et voici le commentaire d'Ernest Havet au fragment XXV.17, qui est notre S. 233 / L. 201. D'abord Havet conseille au lycéen de rapprocher ce texte de deux autres ... qui sont précisément ceux que Valéry citera aussi dans sa Variation de 1923. Puis Havet continue : « Mais ces paroles sont peu de chose auprès de ce grand cri que Port-Royal avait étouffé ». (C'est, en effet, Faugère, en 1844, qui a publié le premier « le silence éternel » ainsi que deux autres fragments à la première personne.) Et une génération plus tard, Brunschvicg dira encore : «Ce cri pénétrant est d'un savant et d'un chrétien ». Et Gide en 1951 - Gide, lui aussi élève de Havet du temps de sa rhétorique - dira à Julien Green : « Dans Pascal, il y a dix cris. On pourrait les citer. Le reste ne m'attire pas. »

En cette fin de siècle où Valéry a grandi, l'angoisse de Pascal est un thème constant. La jeunesse lit Baudelaire, et l'une des Fleurs du mal, que nous avons citée à propos de Proust, commence : 
Pascal avait son gouffre, avec lui se mouvant...

$\mathrm{Tu}$ voyais sous tes pas un gouffre se creuser

Qu'élargissaient sans fin le doute et l'ironie,

Et penché sur cette ombre en ta longue insomnie,

$\mathrm{Tu}$ sentais un frisson mortel te traverser. recherches pascaliennes. ajoutées à la Variation sur une pensée : tourmente et qui m'est odieux. Apologétique :

Tu n'aimes pas ce que tu aimes ;

Tu aimes ce que tu n'aimes pas ;

Tu n'es pas ce que tu es, et réciproquement ${ }^{70}$. Au-dessous d'un portrait :

Que si j'étais placé devant cette effigie

Inconnu de moi-même, ignorant de mes traits,

A tant de plis affreux d'angoisse et d'énergie

Je lirais mes tourments et me reconnaîtrais.

Ceux qui ont eu le privilège de suivre les leçons de Jean Seznec à l'Ashmolean, se souviendront de cette lithographie d'Odilon Redon qui représente un homme épouvanté devant les espaces infinis, que l'artiste a composée en 1878 comme illustration des Pensées , avec le texte du fragment S. 233 / L. 201. En 1880 Jules Lemaître publie dans un recueil de vers, Les Médaillons, un sonnet intitulé Pascal, dont voici le premier quatrain :

On pourrait multiplier les exemples. Quoi d'étonnant que Valéry ait cru, non à l'angoisse de Pascal proprement dite, puisque c'est précisément ce point de la sincérité de Pascal qu'il attaque, mais cru, comme son temps, que Pascal parlait en son propre nom?

Et notez que le malentendu a duré jusqu'à nos jours. Existentialistes et marxistes parlent à l'envi de l'angoisse de Pascal, et la critique érudite ne se met guère en peine de vérifier, surtout quand il s'agit de spécialistes du XVIII ${ }^{e}$ siècle, et la nouvelle critique reprend les reproches de Valéry sans se mettre non plus en peine de s'informer des récentes

Mais c'est précisément ce refus de renoncer à croire à l'angoisse de Pascal qui me fait soupçonner qu'il y a ici une grande part de mauvaise volonté. Cela me permet de revenir à Valéry et de conclure, - au moins provisoirement. « Pascal, l'ennemi intime de Valéry ", a dit Maurice Bémol. Pourquoi ? Valéry nous l'a dit lui-même, il l'a dit dans les notes

Mais c'est l'usage auquel il destinait les fruits amers de sa méditation qui me

Je ne puis souffrir les apologies. S'il est quelque chose qu'un esprit de grande portée se doive interdire, et ne doive même concevoir, c'est l'intention de convaincre les autres et l'emploi de tous les moyens pour y parvenir ${ }^{69}$.

Et dans Mauvaises pensées, dix ans plus tard, se trouve ce petit texte sous le titre;

Voici à quoi sont consacrés les écrits les plus importants :

Ce que Valéry veut défendre à tout prix, c'est sa liberté de penser, une liberté entière, y compris la liberté de ne pas conclure et la liberté de se contredire. Et parce que Pascal en veut à sa liberté, il le combat, et cherche le défaut de la cuirasse dans l'auteur des Pensées. Mais pourquoi cette haine, si ce n'est parce qu'il se sent vulnérable ? Dans le Cahier B 1910 n'a-t-il pas écrit : « Angoisse, mon véritable métier ». Et vingt ans plus tard, ce quatrain

Je ne veux pas prétendre prouver par ces citations que l'angoisse de Valéry soit l'angoisse que Pascal a tant cherché à créer dans son libertin. Je pense qu'elle en est assez différente. Mais je voudrais d'abord conclure sur Valéry, puis sur notre sujet en général.

Sur Valéry, en citant ce qu'on peut lire sur la dernière page du dernier Cahier de Valéry : 
31 mai 1945. Il s'alite pour ne plus se relever.

Juin ou juillet : Pâles au crayon, ses derniers mots écrits :

S« Toutes les chances d'erreur. Pire encore, toutes les chances de mauvais goût, de facilité vulgaire, sont avec celui qui hait. » Et «le mot d'amour ne s'est trouvé associé au nom de Dieu que depuis le Christ. » 20 juillet : Paul Valéry meurt à $9 \mathrm{~h}$ du matin.

Sur notre sujet : même au prix de contresens et de malentendus tels que nous les avons rencontrés, il faut souhaiter que le dialogue avec Pascal continue, car tant que ce dialogue durera, nous pouvons être certains que la vie spirituelle ne sera pas éteinte parmi nous.

\section{NOTES}

1. Sellier 645 / Lafuma 558.

2. S. 38 /L. 2.

3. S. 38 / L. 4.

4. S. 341 / L. 310 .

5. S. 749 / L. 919.

6. S. 748 / L. 918.

7. S. 339 / L. 308.

8. Cf. 560 / L. 681.

9. S. 230 / L. 199.

10. S. 570 / L. 691.

11. Matth. XII, 30.

12. S. 233 / L. 201.

13. S. 102 / L. 68 .

14. S. 681 / L. 427.

15. Rom V, 20.

16. V, 15 .

17. S. 227, 229, 233 / L. 194, 198, 201.

18. Phil. II. 12-13.

19. Matthieu XII, 30.

20. I., 26.

21. II, 22-23.

22. II, 717-718.

23. III, 543.

24. III, 696.

25. III, 728.

26. S. 124 / L. 90.

27. III, 894.

28. L. 681.

29. Port-Royal I, 249.

30. S. 502 / L.606.

31. S. 74 / L. 40.

32. S. 748 / L. 918. 
33. S. 653 / L. 802.

34. S. 552 / L. 673.

35. [S. 773].

36. S. 171 / L. 139.

37. S. 168 / L. 136.

38. S. 348 / L. 317.

39. S. 560 / L. 681.

40. Par ce demi-clair matin, p. 171.

41. A. 171.

42. S. 339 /L. 308. Havet, XIII.1.

43. S. 231 / L. 200 ; Havet, I. 6.

44. A. 1302-1309.

45. Cahiers, $3^{\mathrm{e}}$ série, $14^{\mathrm{e}}$ cahier, 22 avril 1902.

46. Par ce demi-clair matin, p. 169.

47. Par ce demi-clair matin, p. 183 ss.

48. Situation faite à l'histoire et à la sociologie, p. 25-26.

49. S. 680 / L. 418.

50. Halévy, op.cit, p. 241

51. A. 1088

52. A. $774-775$

53. Havet, I. 3 et 4

54. Pléiade, I. 1706

55. S. 74 / L. 40; Havet VII. 31

56. II. 749

57. II. 841

58. II. 417

59. I. 325

60. Havet VI. 20; L. 597

61. Tel quel, Choses tues, II. 499

62. II. 808

63. S. 233 / L. 201.

64. S. 749 / L. 919.

65. S. 227 / L. 194.

66. S. 229 / L. 198.

67. S. 229 / L. 198.

68. II. 126.

69. I. 468.

70. II. 805.

INDEX

Mots-clés : Pascal, dialogue, Proust (Marcel), Péguy (Charles), Valéry (Paul) 
AUTEUR

ANNIE BARNES

Université d'Oxford 